Japan. J. Math.

Vol. 19, No. 1, 1993

\title{
Differential-geometric studies on dynamics of geodesic and frame flows
}

\author{
By Masahiko KanAI \\ (Received April 25, 1991) \\ (Revised January 10, 1992)
}

\section{Introduction}

What we attempt to do in the present article is to understand certain hyperbolic dynamical systems in the language of classical differential geometry. Precisely speaking, the dynamical systems that we are going to deal with are geodesic flows and frame flows of riemannian manifolds of negative curvature, which have also arisen from differential geometry, while the geometric structures in terms of which we are going to analyze these dynamical systems are connections and conformal structures.

To state the results we are going to show, suppose that $M$ is a closed riemannian manifold of negative curvature. As is well known, the geodesic flow $\varphi_{t}$ of the manifold $M$, which is by definition a smooth dynamical system on the unit tangent bundle $V$ of $M$, is an Anosov flow: In other words, the tangent bundle of the phase space $V$ decomposes into three $\varphi_{t}$-invariant continuous subbundles $E^{0}, E^{-}$and $E^{+}$ in such a manner that $E^{0}$ is spanned by the geodesic spray and that every vector in $E^{-}$(resp. $\left.E^{+}\right)$contracts exponentially along an orbit of the geodesic flow in the positive (resp. negative) direction. We call the splitting $T V=E^{-}+E^{0}+E^{+}$ the Anosov splitting, and in what follows we are mainly concerned with the case where the Anosov splitting is $C^{1}$-differentiable. The riemannian manifolds of negative curvature whose geodesic flows carry $C^{1}$-differentiable Anosov splittings form a reasonably broad class. In fact by Green [Gre] and Hirsch-Pugh [HP1], [HP2] there has been obtained a sufficient condition for the Anosov splitting to be $C^{1}$ differentiable. In order to recall their result take two constants $\Lambda \geq \lambda>0$ so that the following inequalities hold for the geodesic flow $\varphi_{t}$ of $M$ with some constant $c>0$ :

$$
\begin{gathered}
c^{-1} e^{-\Lambda t}\left|\xi^{-}\right| \leq\left|d \varphi_{t} \xi^{-}\right| \leq c e^{-\lambda t}\left|\xi^{-}\right| \quad \text { for } \xi^{-} \in E^{-} \text {and } t \geq 0, \\
c^{-1} e^{-\Lambda t}\left|\xi^{+}\right| \leq\left|d \varphi_{-t} \xi^{+}\right| \leq c e^{-\lambda t}\left|\xi^{+}\right| \quad \text { for } \xi^{+} \in E^{+} \text {and } t \geq 0 .
\end{gathered}
$$

For example, if $-\Lambda^{2}$ and $-\lambda^{2}$ are respectively lower and upper bounds of the sec- 
tional curvature of $M$ then it is easy to verify the above inequalities by means of a standard comparison theorem in riemannian geometry. What has been proved by Hirsch, Pugh and Green is that the Anosov splitting is $C^{1}$-differentiable provided that the constants in the inequalities (PIN) satisfy the pinching condition $\Lambda / \lambda<2$. As already mentioned, the last condition is fulfilled e.g. if the sectional curvature $K$ of $M$ satisfies the pinching condition $-4<K \leq-1$. Our geometric investigation of geodesic flows begins with constructing an affine connection on the phase space $V$ of the geodesic flow under the condition that the Anosov splitting is $C^{1}$-differentiable. This will be carried out in $\S 1$, and the connection constructed there will be the most fundamental tool throughout the present article.

The next topic we are going to discuss is the relation between geodesic flows and conformal geometry. In case the manifold $M$ is a hyperbolic manifold (i.e., a riemannian manifold of constant curvature -1 ), the stable bundle $E^{-}$appearing in the Anosov splitting $T V=E^{-}+E^{0}+E^{+}$has a $\varphi_{t}$-invariant conformal structure in a natural way. The most essential part of the proof of Mostow's rigidity theorem [M1], [M2] (see also Sullivan [S1], Thurston [Th]) can be interpreted as the uniqueness of this conformal structure. Besides, Gromov [Gro] has indicated that if the stable bundle $E^{-}$of the geodesic flow of a negatively curved manifold admits a $\varphi_{t}$-invariant conformal structure then the manifold $M$ is homotopic to a hyperbolic manifold. Concerned with this, Sullivan [S2] proposed a conjecture that under the same assumption the manifold $M$ itself is to be of constant curvature. In $\S 2$ we will prove the following theorem, which strengthens Gromov's theorem, and simultaneously supports Sullivan's conjecture.

Theorem 1. Let $\operatorname{dim} M \geq 3$. Suppose either that (a) the stable bundle $E^{-}$ carries a $\varphi_{t}$-invariant continuous conformal structure, or that (b) the constants $\Lambda$ and $\lambda$ in (PIN) satisfy the pinching cndition $\Lambda / \lambda<2$ and $E^{-}$admits a $\varphi_{t}$-invariant bounded measurable conformal structure. Then the geodesic flow of $M$ is homothetic to the geodesic flow of a certain closed hyperbolic manifold $M^{\circ}$.

In the theorem the geodesic flow $\varphi_{t}$ of $M$ is said to be homothetic to the geodesic flow $\varphi_{t}^{\circ}$ of the hyperbolic manifold $M^{\circ}$ in the sense that there are a diffeomorphism $h$ of the unit tangent bundle of $M$ onto that of $M^{\circ}$ and a constant $a>0$ such that $h \circ \varphi_{t}=\varphi_{a t}^{\circ} \circ h$. By virtue of the theorem the conjecture of Sullivan mentioned earlier amounts to the following one which has been already put forward in [K1] in a slightly different context.

CONJECTURE. If the geodesic flow of a closed riemannian manifold $M$ of negative curvature is homothetic to that of a hyperbolic manifold $M^{\circ}$, then $M$ is homothetic to $M^{\circ}$.

Although the conjecture is already known to be true in the case of dimension two by Katok [Kt] (see also Hurder-Katok [HK] for an alternative proof), the 
higher-dimensional case still remains open. Under the assumption of the conjecture, $M$ and $M^{\circ}$ are obviously homotopy equivalent to each other since they have isomorphic fundamental groups, and therefore Mostow's rigidity theorem guarantees that what we have really to do is to show that $M$ is of constant curvature. On the other hand for flows to be homothetic is a global condition, for any two nonsingular flows are always locally isomorphic to each other. Thus the problem is to derive a local consequence from a global assumption.

In $\S 3$ we proceed to a study of the geodesic flows in terms of invariant differential forms. As the geodesic flow is a smooth dynamical system, it induces an action on tensor fields of the phase space. In particular it seems reasonable to expect that invariant tensor fields, or more specifically, invariant differential forms contain some information of the base manifold $M$ as well as the dynamics of the geodesic flow. The purpose of that section is to show that this is in fact the case at least in the following sense. Suppose now that $M$ is a closed riemannian manifold of negative curvature. Since we are mainly interested in "transverse" dynamics of the geodesic flow, it is natural to restrict our attention to "transverse" differential forms. Here a differential form on the unit tangent bundle $V$ of $M$ is said to be transverse (to the geodesic flow) if the the inner product of it with the geodesic spray identically vanishes. Denote by $\Omega_{\varphi_{t}}^{*}(V)$ the graded algebra of $\varphi_{t}$-invariant transverse differential forms on $V$. What we attempt to do in $\S 3$ is to characterize the geodesic flow of a hyperbolic manifold in terms of $\Omega_{\varphi_{t}}^{*}(V)$. More precisely, we will prove

Theorem 2. Suppose that $M$ is a 3-dimensional closed riemannian manifold of negative curvature, and that its geodesic flow satisfies the pinching condition $\Lambda / \lambda<2$. Then the geodesic flow of $M$ is homothetic to the geodesic flow of a certain hyperbolic manifold if and only if $\operatorname{dim} \Omega_{\varphi_{t}}^{2}(V)=2$.

Note that if the conjecture mentioned before is true then the theorem really means that $M$ itself is of constant negative curvature if and only if $\operatorname{dim} \Omega_{\varphi_{t}}^{2}(V)=2$. The "only if" part of the theorem will be shown by computing $\Omega_{\varphi_{t}}^{*}(V)$ explicitly for a hyperbolic manifold in any dimension. Meanwhile the proof of the converse will be done by finding an invariant conformal structure of the stable bundle $E^{-}$ and applying the previous theorem to it. Theorem 2 is an outcome of a suggestion made by A. Katok, and the author would like to express thanks to him.

In the last section we turn to the investigation of frame flows. Suppose now that $M$ is an oriented closed riemannian manifold of negative curvature. The frame flow of $M$ is defined on the space $W$ of the positively oriented orthonormal frames of $M$, which is easily seen to be a principal $\mathrm{SO}(m)$-bundle over the unit tangent bundle $V$ of $M(m+1=\operatorname{dim} M)$, and is a lift of the geodesic flow $\varphi_{t}$ of $M$ through the projection of $W$ onto $V$ (see $\S 4$ for the precise definition of the frame flow). Thus the frame flow of $M$, which we denote by $\psi_{t}$, is a so-called partially hyperbolic dynamical system, or an $\mathrm{SO}(m)$-extension of the geodesic flow $\varphi_{t}$ that is Anosov, 
and it is known that the tangent space of $W$ carries a splitting

$$
T W=F^{v e r}+F^{h o r} \quad \text { with } \quad F^{h o r}=F^{-}+F^{0}+F^{+}
$$

into subbundles satisfying the following conditions: (i) $F^{v e r}$ is vertical with respect to the projection of $W$ onto $V$, while $F^{h o r}$ is horizontal; (ii) $F^{0}$ is 1-dimensional and tangent to the orbits of the frame flow $\psi_{t}$; (iii) any vector in $F^{-}$(resp. $F^{+}$) contracts exponentially along an orbit of $\psi_{t}$ in the positive (resp. negative) direction. The problem we are going to discuss in $\S 4$ is the differentiability of this splitting. In the case of the geodesic flow, it is believed that the higher-order differentiability of the Anosov splitting restricts the structure of the manifold and the geodesic flow to a great degree. In fact in [K1] we have proposed the conjecture that for a closed riemannian manifold of negative curvature the geodesic flow of it has a $C^{2}$-differentiable Anosov splitting if and only if the manifold is locally symmetric. The conjecture has already been verified in the case of dimension two by Ghys [Gh] by using a result of Hurder-Katok [HK], and in the higher dimensions partial results have been obtained by Kanai [K1], Feres-Katok [FK1], [FK2], Feres $[\mathrm{F}]$, Benoit-Foulon-Labrie [BFL] (See also Flaminio-Katok [FlK] for the case of Anosov diffeomorphisms, and Ballmann-Brin-Burns $\left[\mathrm{B}^{3}\right]$ and Hasselblatt [Hs] for examples of Anosov systems whose Anosov splittings have less smoothness). A similar phenomenon is likely to be observed even in the case of frame flows. Actually we would like to propose the following

Conjecture. For a closed riemannian manifold $M$ of negative curvature, the horizontal subbundle $F^{\text {hor }}$ of $T W$ associated with the frame flow of $M$ is $C^{1}$ differentiable if and only if $M$ is locally symmetric.

As a matter of fact we will show the following partial result.

Theorem 3. Take $M$ as in Theorem 2. If the horizontal bundle Fior of the frame flow of $M$ is $C^{2}$-differentiable, then the geodesic flow of $M$ is homothetic to the geodesic flow of a hyperbolic manifold.

The point in the proof of the theorem is that the horizontal subbundle $F^{\text {hor }}$ can be regarded as a connection of the principal bundle $W$ over $V$. Thus if it is smooth, we can speak of the curvature of it. In particular under the assumption of the theorem we will be able to construct a $\varphi_{t}$-invariant differential 2 -form of $V$ from the curvature form of $F^{h o r}$, and prove the theorem by virtue of Theorem 2 .

Acknowledgement. The present work was done while the author was visiting the Department of Mathematics, California Institute of Technology. He wishes to thank the faculty of the department for their hospitality. 


\section{$\S 1$. Affine connections associated with geodesic flows}

The purpose of the present section is to construct an affine connection on the phase space of the geodesic flow. Basically this has already been done in our previous work [K1], [K2], [K3], but we nevertheless repeat it here to explain them in a unified manner as well as for the convenience of the reader. In what follows $\varphi_{t}$ always denotes the geodesic flow of a closed riemannian manifold $M$ of negative curvature defined on the unit tangent bundle $V$ of $M$. Let $T V=E^{-}+E^{0}+E^{+}$be the Anosov splitting attached to the geodesic flow: Namely, $E^{0}$ is the 1-dimensional subbundle of $T V$ generated by the geodesic spray, where the geodesic spray is by definition the nonsingular vector field on $V$ that generates the flow $\varphi_{t}$, while $E^{-}$and $E^{+}$ are respectively the stable and unstable subbundles each of which is characterized by the condition that a vector $\xi^{-} \in E^{-}$(resp. $\xi^{+} \in E^{+}$) contracts exponentially along the orbit of the geodesic flow in the positive (resp. negative) direction. As is well known there are foliations $\mathcal{E}^{-}$and $\mathcal{E}^{+}$of $V$, called the (strongly) stable and unstable foliations, which integrate the stable and unstable subbundles $E^{-}$and $E^{+}$ respectively.

1.1. The ingredients of the affine connection of $V$ which we are going to construct are symplectic geometry of the unit tangent bundle and the hyperbolicity of the geodesic flow. More precisely, the former is a canonically defined 1-form $\theta$ on $V$, called the canonical contact form of $V$. Its exterior derivative $d \theta$ is a symplectic structure on the subbundle $E=E^{-}+E^{+}$of $T V$. In addition the splitting of $E$ into the two parts $E^{-}$and $E^{+}$is easily seen to be a lagrangian splitting with respect to the symplectic structure $d \theta$ of $E$; that is, $d \theta\left(\xi^{-}, \eta^{-}\right)=d \theta\left(\xi^{+}, \eta^{+}\right)=0$ for $\xi^{ \pm}, \eta^{ \pm} \in E^{ \pm}$. Meanwhile the other ingredient is the Anosov splitting itself, or alternatively, the tensor field $f$ on $V$ of type $(1,1)$ that is defined by $f\left(\xi^{-}\right)=-\xi^{-}$, $f\left(\xi^{+}\right)=\xi^{+}$and $f(\dot{\varphi})=0$ for $\xi^{-} \in E^{-}, \xi^{+} \in E^{+}$and the geodesic spray $\dot{\varphi}$. Then it is clear that the tensor field

$$
g(\xi, \eta)=d \theta(\xi, f(\eta))+\theta \otimes \theta(\xi, \eta) \quad(\xi, \eta \in T V)
$$

on $V$ of type $(0,2)$ is symmetric and nondegenerate; that is, $g$ is a pseudo-riemannian metric of $V$.

To proceed further we have to assume that the Anosov splitting $T V=E^{-}+$ $E^{0}+E^{+}$is $C^{1}$-differentiable, or equivalently that the stable and unstable bundles $E^{-}$and $E^{+}$are $C^{1}$-differentiable (Under this assumption we can speak of $C^{1}$ differentiability of the sections of $E^{-}$and $\left.E^{+}\right)$. Then the $(1,1)$-tensor field $f$ which has been defined in terms of the Anosov splitting is $C^{1}$-differentiable, and as a consequence so is the pseudo-riemannian metric $g$. The differentiability of $g$ particularly guarantees the unique existence of an affine connection $\nabla$ on $V$ satisfying the following two conditions: (i) $g$ is parallel with respect to $\nabla$; (ii) the torsion tensor of $\nabla$ is given by $d \theta \otimes \dot{\varphi}$. In fact this can be seen basically in the same way as the 
unique existence of the riemannian connections, and it follows that $\nabla$ is represented by $g$ and its first-order derivative. Hence $\nabla$ should be continuous. In addition $\nabla$ is invariant under the geodesic flow since so are the canonical contact form and the Anosov splitting of which the connection $\nabla$ has been made. Moreover it is easy to see that the Anosov splitting is invariant under the connection $\nabla$; that is, for any section $\xi^{\delta}$ of $E^{\delta}(\delta=0,-,+)$ the covariant derivative $\nabla_{\eta} \xi^{\delta}$ in the direction of any vector field $\eta$ on $V$ is again a section of $E^{\delta}$.

A connection on $V$ which is slightly different from that described here has been introduced in $[\mathrm{K} 2]$. Actually it has been defined to be a unique affine connection on $V$ satisfying the condition (i) above and the condition that (ii') it is torsion-free, instead of (ii). Furthermore in [K2] it was mentioned that this connection would preserve the Anosov splitting as the connection $\nabla$ introduced above does. However this statement is actually false. We have to replace the connection in [K2], $\S 2$ by the connection that has been defined here. Then the original arguments given in [K2] work without any further change. The author would like to thank Renato Feres who kindly pointed out this error to the author.

1.2. What to do next is to give another description of the connection $\nabla$ introduced in the previous subsection, which is essentially based on an idea first employed in [K1]. For this purpose take the universal cover $\widetilde{M}$ of $M$ and its geodesic flow $\widetilde{\varphi}_{t}$ that is defined on the unit tangent bundle $\widetilde{V}$ of $\widetilde{M}$. Denote by $P=\widetilde{V} / \widetilde{\varphi}_{t}$ the orbit space of the geodesic flow $\widetilde{\varphi}_{t}$. To get a topological picture of $P$, let $B$ be the imaginary boundary of the universal cover $\widetilde{M}$ : It is by definition a topological space homeomorphic to the sphere of dimension $\operatorname{dim} M-1$, and each point of it is a point at infinity of $\widetilde{M}$, or more rigorously, an asymptotic class of geodesic rays in $\widetilde{M}$. By the curvature assumption the boundary $B$ of $\widetilde{M}$ possesses the convexity: Namely for any pair of distinct points of $B$ there is a unique geodesic line in $\widetilde{M}$ combining them. But a geodesic line in $\widetilde{M}$ can clearly be identified with an orbit of the geodesic flow $\widetilde{\varphi}_{t}$ of $\widetilde{M}$. Hence there is a natural one-one correspondence of the orbit space $P$ of the geodesic flow with $B \times B \backslash$ (the diagonal set). Furthermore the orbit space $P$ has a natural differentiable structure for which the projection of $\widetilde{V}$ onto $P=\widetilde{V} / \widetilde{\varphi}_{t}$ is smooth. In the meantime it is not hard to see that the two foliations of $P$ which arise from the product structure of $P=B \times B \backslash$ (diagonal) are indeed the pushforwards of the stable and unstable foliations of the geodesic flow $\widetilde{\varphi}_{t}$ through the projection $\widetilde{V} \rightarrow P$. Denote by $\mathcal{F}^{-}$and $\mathcal{F}^{+}$these foliations of $P$, and by $F^{-}$and $F^{+}$ their tangent bundles. It is clear that these subbundles of $T P$ are as smooth as the stable and unstable bundles $E^{-}$and $E^{+}$. Next consider the canonical contact form $\widetilde{\theta}$ of $\widetilde{V}$ and its exterior derivative $d \widetilde{\theta}$. The latter is a $\widetilde{\varphi}_{t}$-invariant 2 -form on $\widetilde{V}$ such that the restriction of it to the sum $\widetilde{E}=\widetilde{E}^{-}+\widetilde{E}^{+}$of the tangent bundles of the stable and unstable foliations is a symplectic structure of $\widetilde{E}$, and that the splitting of $\widetilde{E}$ into $\widetilde{E}^{-}$and $\widetilde{E}^{+}$is a lagrangian splitting. Hence we can push it forward to 
$P$ through the projection $\tilde{V} \rightarrow P$ to get a symplectic form $\omega$ of $P$ for which the splitting $T P=F^{-}+F^{+}$is lagrangian. Finally consider the fundamental group $\Gamma$ of $M$. It acts on the universal cover $\widetilde{M}$ by the deck transformations, and the induced action of it on the unit tangent bundle $\widetilde{V}$ of $\widetilde{M}$ commutes with the geodesic flow $\widetilde{\varphi}_{t}$. Consequently, $\Gamma$ also acts on the orbit space $P=\widetilde{V} / \widetilde{\varphi}_{t}$ differentiably. It is clear that the action preserves the symplectic form $\omega$ and the foliations $\mathcal{F}^{-}$and $\mathcal{F}^{+}$of $P$ mentioned above.

Now assume again that the Anosov splitting of the tangent bundle of $V$ is $C^{1}$ differentiable so as to consider the connection $\nabla$ on $V$ introduced in the previous subsection, and take the lift $\widetilde{\nabla}$ of it to $\widetilde{V}$ through the covering $\widetilde{V} \rightarrow V$. Since $\widetilde{\nabla}$ is $\widetilde{\varphi}_{t^{-}}$ invariant, we can push it forward to the orbit space $P=\widetilde{V} / \widetilde{\varphi}_{t}$ to get a connection $D$ of $P$. It is clear that $D$ is torsion-free since so is $\nabla$ in the transverse direction $E^{-}+E^{+}$, that $D$ preserves the subbundles $F^{-}$and $F^{+}$since the connection $\nabla$ preserves the subbundles $E^{-}$and $E^{+}$, and that $D$ is invariant under the action of the group $\Gamma$ on $P$ since so is $\widetilde{\nabla}$ under the action of $\Gamma$ on $\widetilde{V}$.

Note here that the projection of $P$ onto $B$ that assigns to each $P=\left(b^{-}, b^{+}\right) \in$ $P=B \times B \backslash$ (diagonal) the first factor $b^{-}$gives rise to a $C^{1}$-differentiable structure on the imaginary boundary $B$, since the foliation $\mathcal{F}^{+}$which consists of the fibers of the first-factor projection $P \rightarrow B$ has a $C^{1}$-differentiable tangent bundle according to the differentiability assumption on the Anosov splitting. As a consequence we can think of (continuous) vector fields of $B$, and their horizontal lifts by the projection of $P$ onto $B$, which are by definition sections of the subbundle $F^{-}$of $T P$. Similarly we can consider the projection of $P=B \times B \backslash$ (diagonal) onto the second factor $B$, the differentiable structure of $B$ arising from it and the lifts of vector fields on $B$ to $P$ as sections of $F^{+}$. Note here that these two differentiable structures on $B$ coincide since the involution of $P$ that assigns $\left(b^{+}, b^{-}\right)$to each $\left(b^{-}, b^{+}\right) \in P=B \times B \backslash$ (diagonal) interchanges the foliations $\mathcal{F}^{-}$and $\mathcal{F}^{+}$smoothly. The most important properties of the connection $D$ on $P$ can be stated in the following form:

Lemma 1.1. (1) The leaves of the foliations $\mathcal{F}^{-}$and $\mathcal{F}^{+}$of $P$ are totally geodesic with respect to $D$. (2) The lift of any vector field on $B$ to $P$ as a section of $F^{-}\left(\right.$resp. $\left.F^{+}\right)$by the first-factor (resp. second-factor) projection $P \rightarrow B$ is parallel with respect to $D$ along the foliation $\mathcal{F}^{+}$(resp. $\left.\mathcal{F}^{-}\right)$. (3) The symplectic form $\omega$ of $P$ constructed earlier is parallel with respect to $D$.

Proof. From the construction of the connections $\nabla$ and $D$, it is clear that $D$ is characterized as the Levi-Civita connection of the pseudo-riemannian metric $g=\omega(\cdot, f(\cdot))$ of $P$, where $f$ is the $(1,1)$-tensor field on $P$ defined by $f\left(\xi^{\delta}\right)=\delta \xi^{\delta}$ for $\xi^{\delta} \in F^{\delta}(\delta=-,+)$. Hence we can get an explicit representation of the connection $D$ in terms of $g$, and by means of it we can easily verify the above assertions.

Here is an immediate application of the lemma. In order to state it note first 
that the parallel translation along a curve with respect to the continuous connection $D$ is well defined, since it is described by a first-order linear ordinary differential equation with continuous coefficients, which is as is well known uniquely solvable under an arbitrary initial condition. Hence we can consider the parallel translations with respect to the connection $D$. Recall now that by the second part of the lemma any lift of a vector field on $B$ by the first-factor projection is a section of $F^{-}$which is parallel with respect to $D$ along $\mathcal{F}^{+}$. This especially means that the parallel translation of a vector of $F^{-}$along a curve $c^{+}$contained in a leaf of $\mathcal{F}^{+}$does not depend on the choice of the curve $c^{+}$: It merely depends on the end points of the curve. Then it follows from the last part of the lemma that the parallel translation of a vector in $F^{+}$along the curve $c^{+}$is also independent of the choice of $c^{+}$. (In fact for a frame $\left\{e_{i}^{-}\right\}$of $F^{-}$at the initial point of the curve $c^{+}$, extend it to a parallel frame field along $c^{+}$, and then take a frame field $\left\{e_{i}^{+}\right\}$of $F^{+}$so that $\omega\left(e_{i}^{-}, e_{j}^{+}\right)=\delta_{i j}$. Then the last assertion of the lemma guarantees that $\left\{e_{i}^{+}\right\}$is indeed parallel along $c^{+}$. Clearly $\left\{e_{i}^{+}\right\}$at the terminal point of $c^{+}$is independent of $c^{+}$since so is $\left\{e_{i}^{-}\right\}$.) In consequence it is natural to say that the connection $D$ is flat along the foliation $\mathcal{F}^{+}$even though it is in general impossible to define the curvature of $D$ (Recall that $D$ is only known to be continuous). Similarly we can show that $D$ is flat along $\mathcal{F}^{-}$ as well. In summary,

Corollary 1.2. The connection $D$ of $P$ is flat along the foliations $\mathcal{F}^{-}$and $\mathcal{F}^{+}$(in the sense of parallel translation as above).

Because of the construction of $D$, it also holds that the connection $\nabla$ on $V$ is flat along the stable and unstable foliations $\mathcal{E}^{+}$and $\mathcal{E}^{-}$. The last conclusion is closely related to our previous work [K3].

\section{§2. A conformal structure invariant under the geodesic flow}

Suppose again that $M$ is a closed riemannian manifold of negative sectional curvature, and take its geodesic flow $\varphi_{t}$ defined on the unit tangent bundle $V$ of $M$. Throughout this section assume further that $m+1=\operatorname{dim} M \geq 3$. In addition choose two constants $\Lambda \geq \lambda>0$ so that the inequalities (PIN) in the introduction are fulfilled for these constants. The main purpose of the present section is to prove

Theorem 2.1. Suppose either that (a) the stable bundle $E^{-}$has a $\varphi_{t}$-invariant continuous conformal structure, or that (b) $E^{-}$carries a $\varphi_{t}$-invariant bounded measurable conformal structure and the constants $\Lambda$ and $\lambda$ in (PIN) satisfy the pinching condition $\Lambda / \lambda<2$. Then the geodesic flow of $M$ is homothetic to that of a certain closed hyperbolic manifold.

What is meant by the boundedness of a conformal structure will be explained soon later in $\S 2.1$. 
In [S2] Sullivan has proposed the conjecture that $M$ is to be of constant curvature under the second assumption (b) in our theorem. Thus the theorem reduces his conjecture to ours that has been put forward in the introduction.

2.1. As one of preliminaries for the proof of the theorem we first need to discuss the space of conformal structures. To begin with consider the space $C_{m}$ of (linear) conformal structures on the euclidean space $\mathbb{R}^{m}$ of dimension $m \geq 2$. Here each conformal structure of $\mathbb{R}^{m}$ is considered to be represented by an inner product, and two inner products stand for the same conformal structure provided that they are proportional, or equivalently, conformal to each other. Obviously the general linear group $\mathrm{GL}(m, \mathbb{R})$ acts on $C_{m}$ transitively, and the isotropy subgroup is isomorphic to $\mathrm{CO}(m)=\mathbb{R}^{\times} \cdot \mathrm{SO}(m)$ with $\mathbb{R}^{\times}=\mathbb{R} \backslash\{0\}$ being the product group. As is well known the homogeneous space $C_{m}=\mathrm{GL}(m, \mathbb{R}) / \mathrm{CO}(m)=\mathrm{SL}(m, \mathbb{R}) / \mathrm{SO}(m)$ carries a $\mathrm{GL}(m, \mathbb{R})$-invariant metric for which $C_{m}$ is a riemannian symmetric space of noncompact type.

This $\operatorname{GL}(m, \mathbb{R})$-invariant metric on the space of conformal structures will take a crucial part in the proof of Theorem 2.1. In addition, the metric also plays an advantageous role in the investigation of conformal geometry from the viewpoint of ergodic theory, even though it is not necessary in our present work. This depends on the fact that the center of mass can be defined for any compactly supported probability measure on $C_{m}$. This is possible because the space $C_{m}$ equipped with the metric mentioned above is a simply connected complete riemannian manifold of nonpositive curvature; actually it is known to be a riemannian symmetric space of noncompact type. For instance, for any two points in $C_{m}$ there always exists a unique geodesic segment in $C_{m}$ combining them, and the center of mass of the probability measure equally supported on these two points is the midpoint of the geodesic segment combining them. In a general case the center of mass is defined by means of the distance function of $C_{m}$ and especially its convexity (See Karcher $[\mathrm{Kr}]$ for the rigorous definition of the center of mass). Furthermore since the center of mass is canonically defined in terms of the riemannian structure of $C_{m}$ only, the correspondence that assigns the center of mass to each compactly supported probability measure on $C_{m}$ is equivariant under the isometric action of $\operatorname{GL}(m, \mathbb{R})$ on $C_{m}$. Meanwhile one of the fundamental techniques in ergodic theory is to take an average or mean in order to obtain an invariant object, and the above idea makes it possible to consider the average of conformal structures to get an invariant conformal structure. A similar idea has actually been utilized by Sullivan [S1] to show that a uniformly quasiconformal group action always has an invariant conformal structure. There is also an alternative definition of the "average" of conformal structures introduced by Tukia [Tu].

Now let us return to the study of the geodesic flow. At each point $v$ in the unit tangent bundle $V$, take the fiber of the stable bundle $E^{-}$, consider the space 
of conformal structures on it as before, and denote it by $C_{v} E^{-}$. In consequence we obtain a bundle $C E^{-}$over $V$ whose fiber over $v$ is $C_{v} E^{-}$. The fibers of this bundle have canonically defined metrics as described earlier, and these fiber metrics have the property that any linear isomorphism between two fibers of $E^{-}$induces an isometry between the corresponding fibers of $C E^{-}$. In particular the lift of the geodesic flow $\varphi_{t}$ to $C E^{-}$preserves these metrics. In addition according to the fiber metrics of $C E^{-}$we can speak of the boundedness of a conformal structure of $E^{-}$. To explain it suppose that $\sigma$ is an arbitrary conformal structure of $E^{-}$, or equivalently, a section of the bundle $C E^{-}$. Then it is said to be bounded if at each point $v \in V$ the distance between $\sigma(v)$ and $\sigma_{0}(v)$ in the fiber $C_{v} E^{-}$is bounded by a constant which is independent of the point $v$, where $\sigma_{0}$ is another continuous section of $C E^{-}$. Note that this definition does not depend on the choice of $\sigma_{0}$ because of the compactness of $V$ and the continuity of $\sigma_{0}$.

2.2. We now start the proof of Theorem 2.1. To begin with we have to notice that the assumption (a) in the theorem indeed guarantees the $C^{1}$-differentiability of the Anosov splitting. (This can be seen as follows. First note that the conformal structure on the stable bundle $E^{-}$always induces a conformal structure on its dual bundle $E^{-*}$ in a canonical way. In addition since the direct sum $E=E^{-}+E^{+}$of the stable and unstable bundles carries the symplectic structure $d \theta$ for which the splitting of $E$ into $E^{-}$and $E^{+}$is lagrangian (cf. $\S 1.1$ ), there is a natural linear isomorphism between $E^{-*}$ and $E^{+}$that assigns $\left.\xi^{+}\right\lrcorner d \theta \in E^{-*}$ to each $\xi^{+} \in E^{+}$. Thus under the assumption (a) we consequently have a $\varphi_{t}$-invariant continuous conformal structure on the unstable bundle $E^{+}$as well. Then making use of these continuous conformal structures, we can proceed as if the "strongest" pinching condition $\Lambda=\lambda$ were satisfied in the proof of the theorem of Hirsch-Pugh, and eventually come to the conclusion that the Anosov splitting is $C^{1}$-differentiable.) In short, under either of the assumptions (a) and (b) in Theorem 2.1, the stable and unstable foliations are $C^{1}$-differentiable, and the unit tangent bundle $V$ carries the connection $\nabla$ whose construction has been demonstrated in the previous section. Thus it also follows that the bundle $C E^{-}$of conformal structures possesses the $C^{1}$-differentiability, and that the connection $\nabla$ of $V$ gives rise to a connection on the bundle $C E^{-}$. The connection of $C E^{-}$is also interpreted as follows. For each differentiable curve $c$ in $V$, the parallel translation of vectors in $E^{-}$along $c$ with respect to the connection $\nabla$ is defined as a linear isomorphism between the fibers of $E^{-}$over the end points of the curve $c$. Hence it induces an isometry between the corresponding fibers of $C E^{-}$, which is called the parallel translation of $C E^{-}$ along $c$. Note here that the parallel translation is equivariant with respect to the geodesic flow $\varphi_{t}$ and its lift to the bundle $C E^{-}$since the connection $\nabla$ on $V$ is invariant under the geodesic flow. In what follows a section of $C E^{-}$, or equivalently, a conformal structure of $E^{-}$, is said to be parallel if the parallel translation along 
any curve in $V$ keeps it invariant. It is clear that a parallel conformal structure is $C^{1}$-differentiable since our connection is known to be continuous. The purpose of the present subsection is to show

Claim 2.2. Assume that one of the conditions (a) and (b) in Theorem 2.1 is satisfied. Then any $\varphi_{t}$-invariant bounded measurable conformal structure of $E^{-}$is parallel almost everywhere.

Proof. For any two points $v$ and $w$ sitting in the same leaf $L^{-}$of the stable foliation $\mathcal{E}^{-}$, the parallel translation $\prod_{v, w}^{-}: C_{w} E^{-} \rightarrow C_{v} E^{-}$is defined in such a manner that it is independent of the choice of a curve $c$ along which the translation is considered, provided that $c$ is restricted to be contained in the leaf $L^{-}$. Actually that is a consequence of the flatness of the connection $\nabla$ along $\mathcal{F}^{-}$, which we have established in $\S 1.2$. For a conformal structure $\tau$ of $E^{-}$and a subset $W$ of $V$, we can define the oscillation of $\tau$ restricted to $W$ in the stable direction by

$$
O_{W}^{-}(\tau ; v, r)=\sup _{w \in W \cap B_{r}^{-}(v)} d_{v}\left(\tau(v), \prod_{v, w}^{-} \tau(w)\right) \quad(v \in W, r>0)
$$

where $B_{r}^{-}(v)$ denotes the $r$-ball around $v$ in the leaf of the stable foliation $\mathcal{F}^{-}$ that contains $v$, and $d_{v}$ denotes the metric of the fiber $C_{v} E^{-}$. Recall that the fiber metrics $d_{v}$ are invariant under the lift of the geodesic flow $\varphi_{t}$, that the parallel translation is a $\varphi_{t}$-equivariant isometry, and that the geodesic flow contracts the stable manifolds as one of the inequalities in (PIN) says. As a consequence it follows that

$$
O_{\varphi_{-t} W}^{-}\left(\varphi_{t}^{*} \tau ; v, r\right) \leq O_{W}^{-}\left(\tau ; \varphi_{t} v, c e^{-\lambda t} r\right) \quad\left(v \in \varphi_{-t} W, r, t>0\right)
$$

for any conformal structure $\tau$ and its pull-back $\varphi_{t}^{*} \tau$ by the geodesic flow.

Suppose now that $\sigma$ is a $\varphi_{t}$-invariant bounded measurable conformal structure of $E^{-}$. We can always approximate it by a continuous conformal structure: Actually for any $\varepsilon>0$ we can take a continuous conformal structure $\tau$ of $E^{-}$such that $\mu(V \backslash W)<\varepsilon$ for $W=\left\{v \in V: d_{v}(\sigma(v), \tau(v)) \leq \varepsilon / 3\right\}$, where $\mu$ denotes the Liouville measure of $V$. Note here that $d_{v}\left(\sigma(v),\left(\varphi_{t}^{*} \tau\right)(v)\right)<\varepsilon / 3$ for $v \in \varphi_{-t} W$, since both $d_{v}$ and $\sigma$ are $\varphi_{t}$-invariant. Thus from the definition of the oscillation it follows that

$$
\left|O_{\varphi_{-t} W}^{-}(\sigma ; v, r)-O_{\varphi_{-t} W}^{-}\left(\varphi_{t}^{*} \tau ; v, r\right)\right| \leq \frac{2 \varepsilon}{3}
$$

for $v \in \varphi_{-t} W$. Combining this with the previous inequality we get

$$
O_{\varphi_{-t} W}^{-}(\sigma ; v, r) \leq \frac{2 \varepsilon}{3}+O_{W}^{-}\left(\tau ; \varphi_{t} v, c e^{-\lambda t} r\right)
$$


for $v \in \varphi_{-t} W$ and $r, t>0$. Recall here that the compactness of $V$ implies the absolute continuity of $\tau$. Hence the second term of the right-hand side is less than $\varepsilon / 3$ if $t>0$ is large enough. Take $t$ like this. Then what we have shown so far can be stated as follows: For any $\varepsilon>0$ there is a measurable subset $W_{\varepsilon}=\varphi_{-t} W$ of $V$ such that $\mu\left(V \backslash W_{\varepsilon}\right)<\varepsilon$ and that $O_{W_{\varepsilon}}^{-}(\sigma ; v, r)<\varepsilon$ for $v \in W_{\varepsilon}$. Now put $V_{k}=\bigcap_{j=k}^{\infty} W_{2^{-j}}$ for each positive integer $k$. Then it immediately follows that $\mu\left(V \backslash V_{k}\right)<2^{1-k}$ and that $O_{V_{k}}^{-}(\sigma, v, r)=0$ for $v \in V_{k}$. However this means that $V_{\infty}=\bigcup_{k=1}^{\infty} V_{k}$ is a conull subset of $V$ such that $O_{V_{\infty}}^{-}(\sigma ; v, r)=0$ for all $v \in V_{\infty}$, or equivalently that $\sigma$ is parallel along the stable foliation $\mathcal{F}^{-}$almost everywhere. In the same way we can also show that $\sigma$ is parallel along the unstable foliation $\mathcal{F}^{+}$almost everywhere. Finally we have to show that $\sigma$ is parallel along the orbit of the geodesic flow almost everywhere, but this is trivially satisfied since $\sigma$ is $\varphi_{t}$-invariant. Hence $\sigma$ should be parallel almost everywhere on the entire space $V$. This completes the proof of Claim 2.2 .

As we have seen in the above arguments, our proof of Claim 2.2 is quite similar to the proof of the ergodicity of the geodesic flow given by Anosov $[\mathrm{A}], \S 19$. Actually what we needed in our proof of Claim 2.2 in addition to those which are utilized in the proof of the ergodicity are the $\varphi_{t}$-invariant connection $\nabla$, or alternatively, the $\varphi_{t}$-equivariant parallel translation $\prod_{v, w}^{-}$, and the $\varphi_{t}$-invariant fiber metrics $d_{v}$ of the bundle $C E^{-}$. In terms of them we could define the oscillation that has played the crucial role in our proof.

2.3. The second step of the proof of Theorem 2.1 consists of the study of the imaginary boundary $B$ of the universal cover $\widetilde{M}$ of $M$ and the orbit space $P$ of the geodesic flow $\widetilde{\varphi}_{t}$ of $\widetilde{M}$. Also in this subsection we are going to construct the model space $M^{\circ}$ that has appeared in the theorem. To start with we prove

Claim 2.3. Assume that the condition (a) or (b) in Theorem 2.1 is satisfied. Then the imaginary boundary $B$ possesses a $C^{2}$-differentiable structure for which the first- and second-factor projections of $P=B \times B \backslash$ (diagonal) onto $B$ are $C^{2}$-differentiable. Also the action of the fundamental group $\Gamma$ of $M$ on $B$ is $C^{2}$ differentiable with respect to this differentiable structure. Furthermore $B$ carries a $C^{1}$-differentiable $\Gamma$-invariant flat conformal structure.

In the last statement the conformal structure of $B$ is said to be flat in the sense that $B$ is covered by $\left(C^{2}\right.$-differentiable) charts that transmit the conformal structure of $B$ to the standard one of the euclidean space.

Proof of (Claim 2.3). First we have to construct a conformal structure of the subbundle $F^{-}$of $T P$. To do this note that according to the assumption the stable bundle $E^{-}$carries a continuous or bounded measurable $\varphi_{t}$-invariant conformal structure. Then Claim 2.2 tells us that it should be parallel with respect 
to the connection $\nabla$. Now recall the construction of the connection $D$ on $P$ from $\nabla$ on $V$ described in $\S 1.2$ : This has been done by first taking the lift from $V$ to $\widetilde{V}$ through the covering $\widetilde{V} \rightarrow V$, where $\widetilde{V}$ denotes the unit tangent bundle of the universal cover $\widetilde{M}$ of $M$, and then by pushing forward the lift from $\widetilde{V}$ to $P$ through the projection $\widetilde{V} \rightarrow P$, where $P=\widetilde{V} / \widetilde{\varphi}_{t}$ denotes the orbit space of the geodesic flow $\widetilde{\varphi}_{t}$ of $\widetilde{M}$ defined on $\widetilde{V}$. Similarly first take the lift of the conformal structure of $E^{-} \subset T V$ to the stable subbundle $\widetilde{E}^{-} \subset T \widetilde{V}$ of the geodesic flow $\widetilde{\varphi}_{t}$, which is obviously $\widetilde{\varphi}_{t}$-invariant, and then push it forward to the orbit space $P$ through the projection $\widetilde{V} \rightarrow P$. As a consequence we get a $\Gamma$-invariant conformal structure of the subbundle $F^{-}$of $T P$ that is parallel with respect to the connection $D$ of $P$.

Recall here that under either of the assumptions (a) and (b) in Theorem 2.1 the Anosov splitting of the geodesic flow of $M$ is $C^{1}$-differentiable, and consequently the imaginary boundary $B$ has a $C^{1}$-differentiable structure for which the projections of $P$ onto $B$ as well as the $\Gamma$-action on $B$ are $C^{1}$-differentiable ( $\left.c f . \S 1.2\right)$. In particular we can speak of a conformal structure on $B$. Now the description of the connection $D$ on $P$ mentioned in the second part of Lemma 1.1 implies that the $D$-parallel conformal structure of $F^{-}$should be a lift of a certain conformal structure on $B$ by the first factor projection $\pi^{-}: P \rightarrow B$. Clearly this conformal structure on $B$ is continuous and $\Gamma$-invariant, and therefore we have obtained a weaker version of the claim we are going to prove. In order to explain how to improve the result, take two leaves $L_{0}^{-}$and $L_{1}^{-}$of the foliation $\mathcal{F}^{-}$. Since $F^{-}$is the tangent bundle of the foliation $\mathcal{F}^{-}$, the conformal structure of $F^{-}$gives rise to conformal structures of the leaves $L_{0}^{-}$and $L_{1}^{-}$. Meanwhile restrict the first-factor projection $\pi^{-}: P \rightarrow B$ to these leaves to get two maps $\pi^{-} \mid L_{0}^{-}: L_{0}^{-} \rightarrow B$ and $\pi^{-} \mid L_{1}^{-}: L_{1}^{-} \rightarrow B$. Then the composition $h=\left(\pi^{-} \mid L_{1}^{-}\right)^{-1} \circ\left(\pi^{-} \mid L_{0}^{-}\right)$is a $C^{1}$ conformal diffeomorphism defined on an open subset of $L_{0}^{-}$. To see the existence of a $C^{2}$-differentiable structure of $B$, it is sufficient to show that the partially defined diffeomorphism $h: L_{0}^{-} \rightarrow L_{1}^{-}$is indeed $C^{2}$-differentiable for any $L_{0}^{-}$and $L_{1}^{-}$. Then a $C^{2}$-differentiable structure of $B$ is introduced by regarding the restrictions of the first-factor projection $P \rightarrow B$ to the leaves of $\mathcal{F}^{-}$as $C^{2}$ charts of $B$.

For this purpose we need affine structures of the leaves of the foliation $\mathcal{F}^{-}$. Now take a leaf $L^{-}$of $\mathcal{F}^{-}$. It is already known that $L^{-}$is totally geodesic with respect to the torsion-free affine connection $D$ on $P$ (Lemma 1.1), and the restriction of $D$ to $L^{-}$is flat (Corollary 1.2). By means of the restriction of the connection $D$ to $L^{-}$we can construct "affine" coordinates on $L^{-}$as follows. First pick up a frame of $L^{-}$at a point, and extend it as a parallel frame field: This is possible because of the flatness of the restriction of $D$ to $L^{-}$. Then integrate the parallel vector fields on $L^{-}$of which the frame field consists to obtain a system of flows. These flows commute pairwise since $D$ is torsion-free, and consequently they define a $C^{2}$-differentiable (local) coordinate system of $L^{-}$. The conformal structure of $L^{-}$ seems "constant" in terms of this coordinate system, for the conformal structure 
is parallel with respect to the connection $D$. Thus the leaf $L^{-}$can be (at least locally) identified with the euclidean space equipped with the standard conformal structure via a $C^{2}$ diffeomorphism. Consequently, if two leaves $L_{0}^{-}$and $L_{1}^{-}$of the foliation $\mathcal{F}^{-}$are identified with the euclidean space $\mathbb{R}^{m}$ in this way, then the map $h=\left(\pi^{-} \mid L_{1}^{-}\right) \circ\left(\pi^{-} \mid L_{0}^{-}\right): L_{0}^{-} \rightarrow L_{1}^{-}$introduced earlier is regarded as a $C^{1}$ conformal transformation $h^{\prime}$ defined between open subsets of $\mathbb{R}^{m}$. However it is known that a conformal transformation partially defined on $\mathbb{R}^{m}$ is real-analytic: In fact it is always a restriction of a Möbius transformation whenever $m \geq 3$ (Hartman [Hr]; cf. [M1], [L]), while in the case of $m=2$ it must be holomorphic or antiholomorphic as is well known. Thus it follows that the conformal transformation $h^{\prime}$ on $\mathbb{R}^{m}$ obtained from $h$ is real-analytic, and consequently that $h$ itself is $C^{2}$ differentiable, since the coordinates of $L_{0}^{-}$and $L_{1}^{-}$we have adapted to get $h^{\prime}$ are $C^{2}$-differentiable. This shows the existence of a $C^{2}$-differentiable structure on $B$.

Next we have to prove the $C^{2}$-differentiability of the projections of $P$ onto $B$. To observe the $C^{2}$-differentiability of the first-factor projection $P \rightarrow B$, it is sufficient to show that the derivatives of $h: L_{0}^{-} \rightarrow L_{1}^{-}$, or equivalently of $h^{\prime}: \mathbb{R}^{m} \rightarrow$ $\mathbb{R}^{m}$ up to the second order depend on the leaves $L_{0}^{-}$and $L_{1}^{-}$of $\mathcal{F}^{-}$continuously. Of course $h^{\prime}$ itself depends on $L_{0}^{-}$and $L_{1}^{-}$continuously. On the other hand it is known that the $C^{2}$-norm of any conformal transformation of $\mathbb{R}^{m}(m \geq 2)$ is uniformly bounded by the $C^{0}$-norm of it. Applying this statement especially to $h^{\prime}$, we can conclude the continuous dependence of the derivatives of $h^{\prime}$. Finally to see the $C^{2}$ differentiability of the second-factor projection of $P$ onto $B$, consider the involution $J:\left(b^{-}, b^{+}\right) \mapsto\left(b^{+}, b^{-}\right)$of $P=B \times B \backslash$ (diagonal), which is clearly smooth. Then it satisfies the identity $\pi^{+}=\pi^{-} \cdot J$, where $\pi^{-}$and $\pi^{+}$denote the first and second factor projections respectively. Hence the $C^{2}$-differentiability of $\pi^{+}$is reduced to that of $\pi^{-}$, which has already been known. As a consequence the first- and secondfactor projections of $P$ onto $B$ are both $C^{2}$-differentiable. From this fact it also follows that the action of $\Gamma$ on $B$ is $C^{2}$-differentiable.

Finally we have to check that the conformal structure of $B$ is flat. As we have already seen above, each leaf $L^{-}$of $\mathcal{F}^{-}$is conformally identified with the euclidean space. On the other hand the conformal structure on $B$ has been introduced so that the projection $\pi^{-} \mid L^{-}: L^{-} \rightarrow B$ is conformal. Thus these projections $\pi^{-} \mid L^{-}$ indicate the flatness of the conformal structure of $B$.

The result we have just proved immediately implies the following corollary which was first suggested by Gromov [Gro] without a detailed proof.

COROLlary 2.4. Under the assumptions of Theorem 2.1, M is homotopy equivalent to a certain closed hyperbolic manifold $M^{\circ}$.

Proof. Since the conformal structure of $B$ obtained in the previous claim is flat, $B$ is covered by $C^{2}$ charts that send the conformal structure of $B$ to the canoni- 
cal one of the euclidean space $\mathbb{R}^{m}$. Thus any two charts relate to each other (on their intersection) through a $C^{2}$ conformal diffeomorphism that is defined between domains in the euclidean space. However as we have already seen in the proof of Claim 2.3 , a conformal transformation on the euclidean space is always real-analytic. As a consequence we can apply a standard developing argument to show that the imaginary boundary $B$ of $\widetilde{M}$ is conformally equivalent to the standard sphere $S^{m}$. Hence the conformal action of the fundamental group $\Gamma$ of $M$ on $B=S^{m}$ yields a discrete faithful representation of $\Gamma$ into the conformal transformation group of the standard sphere $S^{m}$, which is known to be canonically isomorphic to Iso $\left(H^{m+1}\right)$, the isometric transformation group of the hyperbolic space $H^{m+1}$. Thus $\Gamma$ turned out to be isomorphic to a discrete subgroup $\Gamma^{\circ}$ of $\operatorname{Iso}\left(H^{m+1}\right)$. The group $\Gamma^{\circ}$ is clearly torsionfree since so is $\Gamma$, and therefore we can form a hyperbolic manifold $M^{\circ}=H^{m+1} / \Gamma^{\circ}$. Then $M$ is homotopy equivalent to $M^{\circ}$ since they are both aspherical manifolds with isomorphic fundamental groups. Finally to see the compactness of $M^{\circ}$ note first that the manifold $M^{\circ}$ is compact if and only if $H_{m+1}\left(M^{\circ} ; \mathbb{Z}_{2}\right) \cong \mathbb{Z}_{2}$. In the meantime $H_{m+1}\left(M ; \mathbb{Z}_{2}\right) \cong \mathbb{Z}_{2}$ holds since $M$ is by assumption compact. Finally we derive an isomorphism $H_{m+1}\left(M ; \mathbb{Z}_{2}\right) \cong H_{m+1}\left(M^{\circ} ; \mathbb{Z}_{2}\right)$ from a homotopy equivalence between $M$ and $M^{\circ}$. This shows the compactness of $M^{\circ}$.

To finish the proof of Theorem 2.1 we must find a closed hyperbolic manifold $M^{\circ}$ whose geodesic flow is homothetic to that of $M$ in the theorem. However we already have a candidate of the model $M^{\circ}$ that was constructed in the previous corollary. What we really have to do for the rest is therefore to find a homothety between the geodesic flow of $M$ and that of $M^{\circ}$, and as a preliminary, we study the symplectic structure of $P$ here.

As to the hyperbolic manifold $M^{\circ}$ instead of $M$, we can speak of all things such as the geodesic flow, the universal cover etc. corresponding to those of $M$. Denote them by attaching ${ }^{\circ}$ at the upper right corner of the symbols representing those constructed from $M$; For example $\varphi_{t}^{\circ}$ denotes the geodesic flow of $M^{\circ}$. Obviously the universal cover $\widetilde{M}^{\circ}$ of $M^{\circ}$ is the hyperbolic space $H^{m+1}$, and its imaginary boundary $B^{\circ}$ is naturally identified with the standard sphere $S^{m}$. Then the $C^{2}-$ differentiable conformal diffeomorphism of $B$ onto $S^{m}$ that we have constructed in the proof of Corollary 2.4 by means of the flat conformal structure of $B$ can be regarded as a $1-1$ correspondence between the imaginary boundaries $B$ and $B^{\circ}$. Furthermore it is equivariant with respect to the actions of the fundamental groups $\Gamma$ of $M$ on $B$ and $\Gamma^{\circ}$ of $M^{\circ}$ on $B^{\circ}$. Let $\iota$ be the natural isomorphism of $\Gamma$ onto $\Gamma^{\circ}$. Then it is reasonable to say that the correspondence between $B$ and $B^{\circ}$ is $\iota$-equivariant. By taking the "product" of the correspondence $B \rightarrow B^{\circ}$ we also have a $C^{2}$-diffeomorphism $f$ of $P=B \times B \backslash$ (diagonal) onto $P^{\circ}=B^{\circ} \times B^{\circ} \backslash$ (diagonal). It is obvious that $f$ is $\iota$-equivariant with respect to the actions of $\Gamma$ on $P$ and $\Gamma^{\circ}$ on $P^{\circ}$, and that $f$ maps the foliations $\mathcal{F}^{-}$and $\mathcal{F}^{+}$of $P$ to those of $P^{\circ}$. In addition 
$f$ preserves the symplectic structures up to a constant: Namely

Claim 2.5. The push-forward of the symplectic form $\omega$ of $P$ by $f$ is proportional to the symplectic form $\omega^{\circ}$ of $P^{\circ}$.

Proof. Recall that the symplectic form $\omega$ of $P$ is $\Gamma$-invariant. Hence the push-forward $f_{*} \omega$ of $\omega$ by $f$ is a $C^{1}$-differentiable $\Gamma^{\circ}$-invariant 2 -form on $P^{\circ}$. Now a computation which will be given in $\S 3.2$ tells us that $f_{*} \omega$ is proportional to $\omega^{\circ}$ provided that $m=\operatorname{dim} M-1 \geq 3$. On the other hand, in the case of $m=2$ we need the exactness. Actually in this case it will be shown in $\S 3.2$ that the $\Gamma^{\circ}$-invariant 2 -forms on $P^{\circ}$ constitute a 2 -dimensional linear space. However it is easy to see that $\omega^{\circ}$ and its constant multiples are the only exact 2 -forms on $P^{\circ}$ that are invariant under the action of $\Gamma^{\circ}$. Meanwhile we can easily show that the canonical symplectic form $\omega$ of $P$ and its push-forward $f_{*} \omega$ are exact. Hence $f_{*} \omega$ should be a constant multiple of $\omega^{\circ}$.

2.4. Here is the last step of the proof of Theorem 2.1 in which we have to construct a homothety between the geodesic flow of $M$ and that of the model space $M^{\circ}$. The arguments developed here are modifications and simplifications of our previous work [K1], $\S \S 4.4-4.5$. First of all notice that the unit tangent bundle $\widetilde{V}$ of the universal cover $\widetilde{M}$ of $M$ can be regarded as a principal $\mathbb{R}$-bundle over the orbit space $P=\widetilde{V} / \widetilde{\varphi}_{t}$ of the geodesic flow $\widetilde{\varphi}_{t}$ of $\widetilde{M}$ : In fact, the action of the structure group $\mathbb{R}$ on $\widetilde{V}$ is given by the geodesic flow $\widetilde{\varphi}_{t}$ itself. In addition the canonical contact form $\widetilde{\theta}$ of $\widetilde{V}$ can be considered to be a connection form of the principal bundle $\widetilde{V} \rightarrow P$. Then the curvature of it is the symplectic form $\omega$ on $P$ (Note here that by the one-dimensionality of the fibers of $\widetilde{V} \rightarrow P$ it is possible to introduce a curvature as a 2 -form on the base space $P$ ). In the same way $\widetilde{V}^{\circ} \rightarrow P^{\circ}$ is considered as a principal $\mathbb{R}$-bundle equipped with a connection $\widetilde{\theta}^{\circ}$. In the meantime, a homothetic change of the metric of the hyperbolic manifold $M^{\circ}$ always changes $\widetilde{\varphi}_{t}^{\circ}, \widetilde{\theta}^{\circ}$, and $\omega^{\circ}$ homothetically. For the convenience, from now on replace the metric of $M^{\circ}$ with a homothetic one so that the diffeomorphism $f: P \rightarrow P^{\circ}$ introduced in the previous subsection preserves the curvature 2-forms $\omega$ on $P$ and $\omega^{\circ}$ on $P^{\circ}$ : Note that this is possible because of (Claim 2.5). Then in order to finish the proof of Theorem 2.1 it is sufficient to show that the diffeomorphism $f$ between the base spaces $P$ and $P^{\circ}$ is lifted to an $\iota$-equivariant diffeomorphism $F: \widetilde{V} \rightarrow \widetilde{V}^{\circ}$ such that $F \cdot \widetilde{\varphi}_{t}=\widetilde{\varphi}_{t}^{\circ} \cdot F$ (i.e., $F$ is an $\iota$-equivariant bundle isomorphism), where $\iota$ is as before the natural isomorphism between the fundamental groups $\Gamma$ of $M$ and $\Gamma^{\circ}$ of $M^{\circ}$ which act on $\widetilde{V}$ and $\widetilde{V}^{\circ}$ respectively: Then $F$ must descend to a homothety $V=\widetilde{V} / \Gamma \rightarrow V^{\circ}=\widetilde{V}^{\circ} / \Gamma^{\circ}$ of the geodesic flows of $M$ and $M^{\circ}$. To begin with we prove

Claim 2.6. The diffeomorphism $f: P \rightarrow P^{\circ}$ is lifted to a bundle isomorphism 
$F: \widetilde{V} \rightarrow \widetilde{V}^{\circ}$ that preserves the connection forms $\widetilde{\theta}$ of $\widetilde{V}$ and $\widetilde{\theta}^{\circ}$ of $\widetilde{V}^{\circ}$. Moreover such a lift is unique in the sense that if both $F_{0}$ and $F_{1}$ are such lifts then there is a constant $T$ such that $F_{1}=\widetilde{\varphi}_{T}^{\circ} \cdot F_{0}$.

Proof. Denote by $W$ the submanifold of the product $\widetilde{V} \times \widetilde{V}^{\circ}$ consisting of all pairs $\left(v, v^{\circ}\right) \in \widetilde{V} \times \widetilde{V}^{\circ}$ such that $f \circ \pi(v)=\pi^{\circ}\left(v^{\circ}\right)$, where $\pi$ (resp. $\pi^{\circ}$ ) denotes the projection of $\widetilde{V}$ (resp. $\widetilde{V}^{\circ}$ ) onto $P$ (resp. $P^{\circ}$ ). Then $W$ naturally carries a structure of principal bundle over $P$ with structure group $\mathbb{R}^{2}$. Let $Z$ be the quotient of $W$ by the restricted action of the diagonal subgroup $\mathbb{R}=\left\{(t, t) \in \mathbb{R}^{2}: t \in \mathbb{R}\right\}$ of $\mathbb{R}^{2}$. Then it is clear that $Z$ is a principal $\mathbb{R}$-bundle over $P$. We can identify a section of $Z \rightarrow P$ with a lift of $f$ to a bundle isomorphism $\widetilde{V} \rightarrow \widetilde{V}^{\circ}$ as follows. Suppose that $F: \widetilde{V} \rightarrow \widetilde{V}^{\circ}$ is a bundle isomorphism that is a lift of $f: P \rightarrow P^{\circ} \dot{\tilde{V}}^{\text {Then at each }}$ point $p$ of $P$ it induces a map $F_{p}: \widetilde{V}_{p} \rightarrow \widetilde{V}_{f(p)}^{\circ}$ between the fibers of $\widetilde{V}$ and $\widetilde{V}^{\circ}$. Now take the graph of $F_{p}$ in $\widetilde{V}_{p} \times \widetilde{V}_{f(p)}^{\circ}$. Here the product $\widetilde{V}_{p} \times \widetilde{V}_{f(p)}^{\circ}$ is by definition the fiber of $W \rightarrow P$ over $p$. In addition the graph of $F_{p}$ in $W_{p}=\widetilde{V}_{p} \times \widetilde{V}_{f(p)}^{\circ}$ is an orbit of the action of the diagonal subgroup $\mathbb{R}$ in $\mathbb{R}^{2}$ on $W$, and therefore is regarded as a point of the fiber of $Z=W / \mathbb{R}$ over $p$. The bundle isomorphism $F: \widetilde{V} \rightarrow \widetilde{V}^{\circ}$ can be always considered as a section of $Z \rightarrow P$ in this manner.

Next we want to introduce a connection on $Z$ as follows. Let $\rho$ (resp. $\rho^{\circ}$ ) be the pull-back of the connection form $\widetilde{\theta}$ (resp. $\widetilde{\theta}^{\circ}$ ) of $\widetilde{V}$ (resp. $\widetilde{V}^{\circ}$ ) by the projection of $\widetilde{V} \times \widetilde{V}^{\circ}$ onto $\widetilde{V}$ (resp. $\left.\widetilde{V}^{\circ}\right)$, and $\sigma$ be the pull-back of $\left(\rho-\rho^{\circ}\right) / 2$ by the inclusion of $W$ into $\widetilde{V} \times \widetilde{V}^{\circ}$. Then $\sigma$ is an $\mathbb{R}^{2}$-invariant 1 -form on $W$, and therefore induces a 1 -form $\tau$ on the quotient $Z=W / \mathbb{R}$, which is easily seen to be a connection form of the principal bundle $Z$ over $P$. Now it is not hard to see that a section of $Z$ is parallel with respect to the connection $\tau$ if and only if the corresponding bundle isomorphism of $\widetilde{V}$ onto $\widetilde{V}^{\circ}$ preserves the connections $\widetilde{\theta}$ of $\widetilde{V}$ and $\widetilde{\theta}^{\circ}$ of $\widetilde{V}^{\circ}$. Recall now that the diffeomorphism $f: P \rightarrow P^{\circ}$ between the base spaces leaves the curvature forms $\omega$ of $\widetilde{\theta}$ and $\omega^{\circ}$ of $\widetilde{\theta}^{\circ}$ invariant, and this especially implies that the connection $\tau$ on $Z$ is flat. In addition $P$ is simply connected since it is homeomorphic to $S^{m} \times S^{m} \backslash$ (diagonal) $(m \geq 2)$. Thus for each $p_{0} \in P$ and a point $z_{0}$ in the fiber of $Z$ over $p_{0}$, there is a unique section $F$ of $Z \rightarrow P$ that is parallel with respect to the connection $\tau$ and satisfies $F\left(p_{0}\right)=z_{0}$. This proves (Claim 2.6).

Now we can improve the previous result into the following form, which immediately implies Theorem 2.1 as we have already observed.

ClaIm 2.7. There exists an $\iota$-equivariant lift $F: \widetilde{V} \rightarrow \tilde{V}^{\circ}$ of $f: P \rightarrow P^{\circ}$ as a bundle isomorphism preserving the connections $\widetilde{\theta}$ of $\widetilde{V}$ and $\widetilde{\theta}^{\circ}$ of $\widetilde{V}^{\circ}$.

Proof. Denote by $I$ the involution of the unit tangent bundle $\widetilde{V}$ that assigns $-v$ to each unit tangent vector $v$ of $\widetilde{M}$. Similarly let $I^{\circ}$ be the involution of $\widetilde{V}^{\circ}$. First we want to show that a lift $F: \widetilde{V} \rightarrow \widetilde{V}^{\circ}$ of $f: P \rightarrow P^{\circ}$ as a connection-preserving 
bundle isomorphism is $\iota$-equivariant provided that it satisfies

$$
F \circ I=I^{\circ} \circ F
$$

It is known that for each nontrivial element $\gamma$ of $\Gamma$ there is a unique geodesic line $l$ in the universal cover $\widetilde{M}$ that is kept invariant under the action of $\gamma$ on $\widetilde{M}$ as a deck transformation (see [EO]). If $v \in \widetilde{V}$ is a vector tangent to $l$ it satisfies

$$
\gamma(v)=\widetilde{\varphi}_{a}(v), \quad \gamma \circ I(v)=\widetilde{\varphi}_{-a} \circ I(v)
$$

with a suitable constant $a$, where in the left-hand sides $\gamma$ denotes the action of $\gamma$ on $\widetilde{V}$ obtained by differentiating the deck transformation $\gamma$ of $\widetilde{M}$. Meanwhile as in $\S 1.2$ every geodesic line in the universal cover $\widetilde{M}$ is canonically identified with a point of $P=B \times B \backslash$ (diagonal), where $B$ denotes the sphere at infinity of $\widetilde{M}$. Moreover $\gamma \in \Gamma$ leaves a geodesic line in $\widetilde{M}$ invariant if and only if the corresponding point of $P$ is a fixed point of the action of $\gamma$ on $P$. Thus it follows that $v^{\circ}=F(v) \in V^{\circ}$ is tangent to a geodesic line in $M^{\circ}$ that is invariant under the deck action of $\gamma^{\circ}=\iota(\gamma) \in \Gamma^{\circ}$ on $M^{\circ}$. Consequently, as before we have

$$
\gamma^{\circ}\left(v^{\circ}\right)=\widetilde{\varphi}_{a^{\circ}}^{\circ}\left(v^{\circ}\right), \quad \gamma^{\circ} \circ I^{\circ}\left(v^{\circ}\right)=\widetilde{\varphi}_{-a^{\circ}}^{\circ} \circ I^{\circ}\left(v^{\circ}\right)
$$

with a certain constant $a^{\circ}$. On the other hand, note that the uniqueness part of the previous claim implies that for each $\gamma \in \Gamma$ and $\gamma^{\circ}=\iota(\gamma) \in \Gamma^{\circ}$ there is a constant $T=T(\gamma)$ depending only on $\gamma$ such that

$$
F \circ \gamma=\widetilde{\varphi}_{T}^{\circ} \circ \gamma^{\circ} \circ F
$$

Thus to show that $F$ is $\iota$-equivariant it is enough to indicate $T=T(\gamma)=0$ for any $\gamma \in \Gamma$. To see this take $v \in \widetilde{V}$ and $v^{\circ}=F(v) \in \widetilde{V}^{\circ}$ so that they satisfy (2.9) and (2.10) as before. By the choice of $T$ we have $F \circ \gamma(v)=\widetilde{\varphi}_{T}^{\circ} \circ \gamma^{\circ}\left(v^{\circ}\right)$. With regard to the left-hand side we have $F \circ \gamma(v)=F \circ \widetilde{\varphi}_{a}(v)=\widetilde{\varphi}_{a}^{\circ}\left(v^{\circ}\right)$ because of the first identify in (2.9) and the fact that $F$ commutes with the geodesic flows $\tilde{\varphi}_{t}$ and $\widetilde{\varphi}_{t}^{\circ}$. Meanwhile by means of the first identity of (2.10) the right hand side is deformed like $\widetilde{\varphi}_{T}^{\circ} \circ \gamma^{\circ}\left(v^{\circ}\right)=\widetilde{\varphi}_{T+a}^{\circ} \circ\left(v^{\circ}\right)$. Thus it follows that $\widetilde{\varphi}_{a}^{\circ}\left(v^{\circ}\right)=\widetilde{\varphi}_{T+a}^{\circ} \circ\left(v^{\circ}\right)$. However this holds only if $a=T+a^{\circ}$. Next we want to show that $-a=T-a^{\circ}$ which immediately implies that $T=0$. First again by the choice of $T$ we get $F \circ \gamma \circ I(v)=\widetilde{\varphi}_{T}^{\circ} \circ \gamma^{\circ} \circ F \circ I(v)$. The left-hand side is rewritten into $\widetilde{\varphi}_{-a}^{\circ} \circ F \circ I(v)$ as before by means of the latter of the two identities in (2.9). Then the assumption (2.8) says that $\widetilde{\varphi}_{-a}^{\circ} \circ F \circ I(v)=\widetilde{\varphi}_{-a}^{\circ} \circ I^{\circ}\left(v^{\circ}\right)$. On the other hand because of $(2.8)$ and $(2.10)$ we have $\widetilde{\varphi}_{T}^{\circ} \circ \gamma^{\circ} \circ F \circ I(v)=\widetilde{\varphi}_{T-a^{\circ}}^{\circ} \circ I^{\circ}\left(v^{\circ}\right)$. As a consequence we obtain $\widetilde{\varphi}_{-a}^{\circ} \circ I^{\circ}\left(v^{\circ}\right)=\widetilde{\varphi}_{T-a^{\circ}}^{\circ} \circ I^{\circ}\left(v^{\circ}\right)$ and $-a=T-a^{\circ}$. This shows that $T=T(\gamma)=0$ for each $\gamma \in \Gamma$, and eventually that $F$ is $\iota$-equivariant whenever it fulfills the condition (2.8). 
What we have seen so far reduces the proof of Claim 2.7 to lifting $f: P \rightarrow P^{\circ}$ to a connection-preserving bundle isomorphism $F: \widetilde{V} \rightarrow \widetilde{V}^{\circ}$ which simultaneously satisfies the condition (2.8). To do this, take a lift $F$ of $f$ as in Claim 2.6. Then it is easy to see that $I^{\circ} \circ F \circ I: \widetilde{V} \rightarrow \widetilde{V}^{\circ}$ is a connection-preserving bundle isomorphism. On the other hand, if $J$ denotes the involution of $P$ that assigns $\left(b^{+}, b^{-}\right)$to each $\left(b^{-}, b^{+}\right) \in P=B \times B \backslash$ (diagonal) then $I$ is a lift of $J$ via the projection of $V$ onto $P$. Similarly $I^{\circ}$ is a lift of the involution $J^{\circ}$ of $P^{\circ}$, and the diffeomorphism $f: P \rightarrow P^{\circ}$ obviously satisfies $f \circ J=J^{\circ} \circ f$. Hence it follows that $I^{\circ} \circ F \circ I$ is also a lift of $f$. Now by the uniqueness part of (Claim 2.6) we can find a constant $T$ such that $I^{\circ} \circ F \circ I=\widetilde{\varphi}_{T}^{\circ} \circ F$. Now replace $F$ with the composite $\widetilde{\varphi}_{T / 2}^{\circ} \circ F$. Then it is clear that the new isomorphism $F$ indeed satisfies (2.8) since the involution $I^{\circ}$ of $V^{\circ}$ satisfies $I^{\circ} \circ \widetilde{\varphi}_{t}^{\circ}=\widetilde{\varphi}_{-t}^{\circ} \circ I^{\circ}$ for any $t$.

\section{§3. Invariant differential forms and conformal structures}

As the geodesic flow is a smooth dynamical system, it is meaningful to consider tensor fields which are invariant under the geodesic flow. In particular what we intend to investigate in this section is the relation between invariant differential forms and conformal geometry. As before let $\varphi_{t}$ denote the geodesic flow of a closed riemannian manifold $M$ of negative curvature defined on the unit tangent bundle $V$ of $M$. Moreover take two constants $\Lambda \geq \lambda>0$ so that the geodesic flow $\varphi_{t}$ satisfies the inequalities (PIN) given in the introduction. On the other hand denote by $\Omega_{\varphi_{t}}^{k}(V)$ the space of $C^{1}$-differentiable transverse $k$-forms of $V$ which are invariant under the geodesic flow $\varphi_{t}$ : Here a differential form $\alpha$ on the unit tangent bundle $V$ is said to be transverse (relative to the geodesic flow) if the inner product $\dot{\varphi}\lrcorner \alpha$ of $\alpha$ with the geodesic spray $\dot{\varphi}$ identically vanishes. If $\theta$ denotes the canonical contact form of $V$ as before, then it is clear that $\omega=d \theta$ is a nonzero element of $\Omega_{\varphi_{t}}^{2}(V)$. Hence the dimension of $\Omega_{\varphi_{t}}^{2}(M)$ is at least one. Now the main result of the present section can be stated in the following form.

THEOREM 3.1. Let $M$ be a 3-dimensional closed riemannian manifold of negative curvature whose geodesic flow $\varphi_{t}$ satisfies the pinching condition $\Lambda / \lambda<2$. Then the geodesic flow of $M$ is homothetic to the geodesic flow of a closed hyperbolic manifold provided that $\operatorname{dim} \Omega_{\varphi_{t}}^{2}(V) \geq 2$.

To begin with we should make a couple of remarks. First of all, we do not know whether the $C^{1}$-differentiability of invariant forms or the pinching condition $\Lambda / \lambda<2$ is really necessary for the theorem. It might be possible that the theorem remains valid even under the weaker assumptions of negativity of curvature and of continuity of invariant differential forms. Secondly the computation of $\Omega_{\varphi_{t}}^{*}(V)$ for hyperbolic manifolds which will be done later in $\S 3.2$ suggests a generalization of the theorem for higher dimensional manifolds. In fact it seems reasonable to 
expect that the same conclusion holds if the manifold $M$ under consideration is $(2 n+1)$-dimensional and if $\operatorname{dim} \Omega_{\varphi_{t}}^{2 n}(V) \geq 2$. However our proof of the theorem is based upon two-dimensional conformal geometry (actually it will be proved by means of Theorem 2.1 that has been obtained in the previous section), and as is well known two-dimensional conformal geometry is very peculiar compared with the higher dimensional cases. So our proof given here cannot be generalized directly to higher-dimensional manifolds, to say the least.

The construction of the present section is as follows. First we want to compute $\Omega_{\varphi_{t}}^{*}(V)$ specifically for hyperbolic manifolds, since the computation supports the plausibility of Theorem 3.1, and in addition, suggests the possibility of the generalization of it to higher dimensional manifolds. Furthermore the computation of $\Omega_{\varphi_{t}}^{*}(V)$ for hyperbolic manifolds clarifies the relation between $\Omega_{\varphi_{t}}^{*}(V)$ and conformal geometry. As a preliminary for doing this, we will show a lemma in $\S 3.1$ which indicates a quite strong restriction on $\Omega_{\varphi_{t}}^{*}(V)$ for "almost" hyperbolic manifolds. Applying it especially for hyperbolic manifolds, we will compute the dimension of $\Omega_{\varphi_{t}}^{*}(V)$ in $\S 3.2$. The proof of Theorem 3.1 will be given in the last subsection $\S 3.3$.

3.1. First of all we must study an effect brought on by a pinching condition on invariant differential forms, or more generally invariant tensor fields. To state the result we first have to generalize the transversality of differential forms to that of tensor fields. Suppose that $\tau$ is a tensor field on $V$ of type $(r, s)$; i.e., $\tau$ is a section of $\left(\otimes_{r} T V\right) \otimes\left(\bigotimes_{s} T^{*} V\right)$. Notice here that the Anosov splitting $T V=E^{-}+E^{0}+E^{+}$ yields the splitting $T^{*} V=E^{-*}+E^{0 *}+E^{+*}$ of the cotangent bundle as well. Hence the bundle of $(r, s)$-tensors also splits accordingly. Then $\tau$ is said to be transverse provided that it has neither $E^{0}$ - nor $E^{0 *}$-component. Obviously this definition is a generalization of that for differential forms. The following result has been first utilized in [K1] implicitly, and stated and proved in [K2] (Also some generalizations have been given by Feres and Katok in [FK1], [FK2], [F]). Note below that the pinching condition $\Lambda / \lambda<2$ is always satisfied, and as a consequence that the unit tangent bundle $V$ carries the $\varphi_{t}$-invariant connection $\nabla$ that has been constructed in $\S 1$.

LEMMA 3.2. Assume that the constants $\Lambda$ and $\lambda$ in (PIN) satisfy the pinching condition $\Lambda / \lambda<(n+1) / n$ with a positive integer $n$. Then (i) any $\varphi_{t}$-invariant continuous transverse tensor field on $V$ of degree $2 k+1 \leq 2 n+1$ identically vanishes. On the other hand, (ii) $\nabla \tau=0$ for any $\varphi_{t}$-invariant $C^{1}$-differentiable transverse tensor field $\tau$ of degree $2 k \leq 2 n$.

Actually the first part is an immediate consequence of the fact that the induced action of the geodesic flow on the bundle $\left(\otimes_{i} E^{-}\right) \otimes\left(\bigotimes_{j} E^{+}\right) \otimes\left(\bigotimes_{j *} E^{-*}\right) \otimes$ $\left(\otimes_{i *} E^{+*}\right)\left(i+j+i^{*}+j^{*}=2 k+1\right)$ is fiberwise uniformly contracting or expanding according to whether $i+i^{*}>j+j^{*}$ or $i+i^{*}<j+j^{*}$. This implies the non-existence 
of $\varphi_{t}$-invariant continuous sections of the bundle other than the zero section. The second assertion of the lemma is obtained just by applying the first to the covariant derivative $\nabla \tau$. See $[\mathrm{K} 2]$ for more details.

Here is a slightly different formulation of Lemma 3.2 together with an implication of Theorem 3.1. As in $\S 1.2$, denote by $P$ the orbit space of the geodesic flow of the universal cover of $M$ on which the fundamental group $\Gamma$ of $M$ acts differentiably. What we want to see first is tensor fields on $P$ that are $\Gamma$-invariant. For each $\Gamma$-invariant tensor field on $P$, first take the lift of it to the unit tangent bundle $\widetilde{V}$ of the universal cover of $M$ through the projection $\widetilde{V} \rightarrow P$. The lift is obviously invariant under the deck transformations of the covering $\widetilde{V} \rightarrow V$, and therefore descends to a tensor field on $V$ which is easily seen to be $\varphi_{t}$-invariant and transverse. Thus Lemma 3.2 implies the following conclusion under the same assumption as in the lemma: (i) $A \Gamma$-invariant continuous tensor field on $P$ of degree $2 k+1 \leq 2 n+1$ vanishes on $P$ : (ii) if $\tau$ is a $\Gamma$-invariant $C^{1}$-differentiable tensor field on $P$ of degree $2 k \leq 2 n$ then $D \tau=0$ for the connection $D$ on $P$ defined in $\S 1.2$.

Now let $\Omega_{\Gamma}^{*}(P)$ be the graded algebra consisting of $\Gamma$-invariant smooth differential forms on $P$. Note that $\Omega_{\Gamma}^{*}(P)$ is a subcomplex of the de Rham cochain complex of $P$. Hence we can think of the cohomology $H^{*}\left(\Omega_{\Gamma}^{*}(P)\right)$ of it. Suppose now that $M$ is "almost hyperbolic", or equivalently that $\Lambda / \lambda$ is sufficiently close to 1. Then as we have just seen, $\Omega_{\Gamma}^{\text {odd }}(P)=0$, and consequently $H^{*}\left(\Omega_{\Gamma}^{*}(P)\right)=\Omega_{\Gamma}^{*}(P)$. In addition there is a natural correspondence between $\Omega_{\Gamma}^{*}(P)$ and $\Omega_{\varphi_{t}}^{*}(V)$ as we already know. Hence the condition in Theorem 3.1 on $\Omega_{\varphi_{t}}^{*}(V)$ can be interpreted as a condition on the cohomology $H^{*}\left(\Omega_{\Gamma}^{*}(P)\right)$. In fact if the conjecture mentioned in the introduction is valid, the theorem says that the hyperbolic manifolds can be characterized by the condition that $H^{2}\left(\Omega_{\Gamma}^{*}(P)\right)=2$ among the almost hyperbolic 3-manifolds. More or less the theorem tells us that $H^{2}\left(\Omega_{\Gamma}^{*}(P)\right)$ is a geometric invariant for negatively curved manifolds. On the other hand it is well known that the action of the fundamental group $\Gamma$ of $M$ on the orbit space $P$ of the geodesic flow of the universal cover of $M$ is uniquely determined by $\Gamma$ up to topological conjugacy. In other words the $\Gamma$-action on $P$ is a topological invariant of $M$ up to topological conjugacy. Hence Theorem 3.1 suggests that the cohomology $H^{*}\left(\Omega_{\Gamma}^{*}(P)\right)$ of invariant differential forms is neither an invariant of topological conjugacy of the $\Gamma$-action on $P$ nor a topological invariant of $M$.

3.2. What we are going to do next is to compute the dimension of $\Omega_{\varphi_{t}}^{*}(V)$ particularly for hyperbolic manifolds. Throughout this subsection let $M$ be a closed orientable hyperbolic manifold of dimension $m+1$, and consider the geodesic flow $\varphi_{t}$ of $M$ defined on the unit tangent bundle $V$ of $M$. Then we have 
Claim 3.3. In the case of $\operatorname{dim} M=2 n+2$,

$$
\operatorname{dim} \Omega_{\varphi_{t}}^{k}(V)= \begin{cases}0 & \text { if } k \text { is odd } \\ 1 & \text { if } k \text { is even and } \leq 4 n+2 .\end{cases}
$$

On the other hand in the case of $\operatorname{dim} M=2 n+1$,

$$
\operatorname{dim} \Omega_{\varphi_{t}}^{k}(V)= \begin{cases}0 & \text { if } k \text { is odd } \\ 1 & \text { if } k \text { is even }, \leq 4 n \text { and } \neq 2 n \\ 2 & \text { if } k=2 n .\end{cases}
$$

To see this we first want to find generators of $\Omega_{\varphi_{t}}^{k}(V)$. Notice that the exterior products $\omega^{k}(k=0, \ldots, m)$ of $\omega=d \theta$, where $\theta$ denotes the canonical contact form as before, are respectively generators of $\Omega_{\varphi_{t}}^{2 k}(V)$. Hence what we have to do is to find another generator of $\Omega_{\varphi_{t}}^{2 n}(V)$ in the case of $\operatorname{dim} M=2 n+1$ so as to explain the 2-dimensionality of $\Omega_{\varphi_{t}}^{2 n}(V)$. For this purpose note first that $\alpha\left(\xi_{1}, \ldots, \xi_{2 n}\right)$ vanishes for $\alpha \in \Omega_{\varphi_{t}}^{2 n}(V)$ and $\xi_{i} \in E^{ \pm}$unless exactly $n$ of $\xi_{i}$ 's are vectors of $E^{-}$and the others are of $E^{+}$: Actually this can be seen as in the proof of Lemma 3.2. Thus in order to determine $\alpha \in \Omega_{\varphi_{t}}^{2 n}(V)$ it is sufficient to specify the coupling $\left\langle\alpha, \xi^{-} \wedge \eta^{+}\right\rangle$ of $\xi^{-} \in \bigwedge^{n} E^{-}, \eta^{+} \in \bigwedge^{n} E^{+}$and $\alpha$. Now recall that the stable bundle $E^{-}$has a natural conformal structure which is invariant under the geodesic flow $\varphi_{t}$, since $M$ is of constant curvature. On the other hand it is known that Hodge's *-operator of the middle-dimensional exterior algebra of the even-dimensional euclidean space $\mathbb{R}^{2 n}$ is a conformal invariant (More precisely $*$ is a linear operator of $\bigwedge^{n} \mathbb{R}^{2 n}$ onto itself which assigs $(\operatorname{sgn} \pi) \cdot e_{\pi_{n+1}} \wedge \cdots \wedge e_{\pi_{2 n}}$ to each $e_{\pi_{1}} \wedge \cdots \wedge e_{\pi_{n}}$, where $\left\{e_{1}, \ldots, e_{2 n}\right\}$ denotes the standard orthonormal basis of $\mathbb{R}^{2 n}$ and $\left(\pi_{1}, \ldots, \pi_{2 n}\right)$ is an arbitrary permutation of $(1, \ldots, 2 n))$. Hence by means of the $\varphi_{t}$-invariant conformal structure of the stable subbundle $E^{-}$we can define Hodge's operator $*$ as a $\varphi_{t}$-invariant smooth section of $\left(\wedge^{n} E^{-}\right)^{*} \otimes\left(\wedge^{n} E^{-}\right)$. Now define an element $\alpha$ of $\Omega_{\varphi_{t}}^{2 n}(V)$ by $\left\langle\alpha, \xi^{-} \wedge \eta^{+}\right\rangle=\left\langle\omega^{n}, *\left(\xi^{-}\right) \wedge \eta^{*}\right\rangle$. Then $\alpha$ is actually linearly independent of $\omega^{n}$.

To complete the proof of Claim 3.3 it is sufficient to show that the invariant differential forms exhibited above indeed $\operatorname{span} \Omega_{\varphi_{t}}^{*}(V)$. For this purpose first note that the universal cover of $M$ is the hyperbolic space $H^{m+1}$. Form the orbit space $P$ of the geodesic flow of the universal cover $H^{m+1}$ as before. Then as we have seen in the previous subsection $\Omega_{\varphi_{t}}^{*}(V)$ is naturally isomorphic to $\Omega_{\Gamma}^{*}(P)$, the space of $\Gamma$-invariant differential forms on $P$. Furthermore in the present case the isometry group Iso $\left(H^{m+1}\right)$ of $H^{m+1}$ acts on $P$ in a natural way, and the restricted action of the identity component $G$ of $\operatorname{Iso}\left(H^{m+1}\right)$ on $P$ is transitive. Since $G$ contains $\Gamma$ as a subgroup of it $\Omega_{G}^{*}(P)$ is embedded in $\Omega_{\Gamma}^{*}(P)$, where the former denotes the graded algebra of $G$-invariant differential forms on $P$. To see the opposite inclusion note 
first that Lemma 3.2 especially applied to the hyperbolic manifold $M$ implies that each element of $\Omega_{\Gamma}^{*}(P)$ is parallel with respect to the connection $D$ on $P$ constructed in $\S 1.2$. In addition, in this case the connection $D$ has another characterization as follows (cf. [K2]): A differential form on $P$ is parallel with respect to $D$ if and only if it is $G$-invariant. Hence by Lemma 3.2 each element of $\Omega_{\Gamma}^{*}(P)$ is also $G$-invariant; namely $\Omega_{\Gamma}^{*}(P) \subset \Omega_{G}^{*}(P)$. Thus we have obtained isomorphisms $\Omega_{\varphi_{t}}^{*}(V) \cong \Omega_{\Gamma}^{*}(P) \cong$ $\Omega_{G}^{*}(P)$. To proceed further recall now that the Lie group $G=\mathrm{SO}_{0}(m+1,1)$ acts on $P$ transitively; i.e., $P$ is a homogeneous $G$-space $G / H$, where $H \cong \mathrm{CO}_{0}(m) \cong$ $\mathbb{R}^{+} \cdot \mathrm{SO}(m)$ (the direct product) is a closed subgroup of $G$. Moreover in this case $P$ is a reductive homogeneous space: Namely the Lie algebra $\mathfrak{g}$ of $G$ has an $\operatorname{Ad}(H)$ invariant linear subspace $\mathfrak{p}$ such that $\mathfrak{g}=\mathfrak{h}+\mathfrak{p}$ (direct sum), where $\mathfrak{h}$ is the Lie algebra of $H$. Clearly $\Omega_{G}^{*}(P)$ is isomorphic to $\bigwedge_{\operatorname{Ad}(H)}^{*}\left(\mathfrak{p}^{*}\right)$, the space of $\operatorname{Ad}(H)$ invariant exterior forms on $\mathfrak{p}$. In summary we have an isomorphism

$$
\Omega_{\varphi_{t}}^{*}(V) \cong \bigwedge_{\operatorname{Ad}(H)}^{*}\left(\mathfrak{p}^{*}\right)
$$

To compute the right hand side, we have to exhibit the adjoint representation of $H$ on $\mathfrak{p}$ explicitly. Actually it is given by $\operatorname{Ad}(h)\left(\xi^{-}, \eta^{+}\right)=\left(\xi^{-} h^{-1}, h \eta^{+}\right)$for $h \in H=\mathrm{CO}_{0}(m)$ and $\left(\xi^{-}, \eta^{+}\right) \in \mathbb{R}^{m} \times \mathbb{R}^{m}=\mathfrak{p}$, where $\xi^{-}$and $\eta^{+}$are regarded as row and column vectors respectively. Now by looking at this representation carefully, we can show that the elements of $\bigwedge_{\mathrm{Ad}(H)}^{*}\left(\mathfrak{p}^{*}\right)$ that correspond to the generators $\omega^{k}(k=0, \ldots, m)$ and $\alpha$ of $\Omega_{\varphi_{t}}^{*}(V)$ described earlier indeed generate $\bigwedge_{\mathrm{Ad}(H)}^{*}\left(\mathfrak{p}^{*}\right)$ linearly. This proves Claim 3.3.

3.3. What we have observed in the last subsection is that it is the conformal structure on the stable bundle $E^{-}$that causes the multiplicity in $\Omega_{\varphi_{t}}^{2 n}(V)$ in the case of a hyperbolic manifold of dimension $2 n+1$. Conversely at least if $M$ is 3 dimensional and if the geodesic flow satisfies the pinching condition $\Lambda / \lambda<2$, then we can recover the conformal structure of the stable bundle from the multiplicity in $\Omega_{\varphi_{t}}^{2}(V)$ as we will soon see later, and this leads us to Theorem 3.1 via Theorem 2.1 obtained in the last section. In the proof we will make use of the special property of two-dimensional conformal geometry. Roughly speaking in dimension two, $($ conformal structure $)+($ orientation $)=($ almost complex structure $)=($ Hodge's *-operator). In the case of $\operatorname{dim} M=3$ the stable bundle $E^{-}$is 2-dimensional, and therefore it is enough to find a $\varphi_{t}$-invariant almost complex structure on it.

In what follows suppose that $M$ is a 3 -dimensional closed riemannian manifold of negative curvature whose geodesic flow $\varphi_{t}$ satisfies the pinching condition $\Lambda / \lambda<$ 2 , and assume especially that the condition $\operatorname{dim} \Omega_{\varphi_{t}}^{2}(V) \geq 2$ is satisfied. Of course the exterior derivative $\omega=d \theta$ of the canonical contact form $\theta$ is a nonzero element of $\Omega_{\varphi_{t}}^{2}(V)$. Take another element $\alpha$ of $\Omega_{\varphi_{t}}^{2}(V)$ that is linearly independent of $\omega$. Then we can define a section $J$ of $E^{-*} \otimes E^{-}$by 


$$
\omega\left(J \xi^{-}, \eta^{+}\right)=\alpha\left(\xi^{-}, \eta^{+}\right) \quad \text { for } \quad \xi^{-} \in E^{-} \quad \text { and } \quad \eta^{+} \in E^{+} .
$$

Note here that the above equation actually defines the operator $J$, since $\omega$ restricted to the direct sum $E=E^{-}+E^{+}$is a symplectic structure of $E$, and the splitting of $E$ into $E^{-}$and $E^{+}$is a lagrangian splitting. What we are going to show in the rest is that $J$ is an almost complex structure of $E^{-}$(i.e., $J^{2}=-1$ ) if $\alpha$ is chosen suitably. First of all note that the pinching condition $\Lambda / \lambda<2$ implies that $V$ has a canonical $\varphi_{t}$-invariant connection $\nabla$, and that $J$ is parallel with respect to it since so are $\omega$ and $\alpha$ by Lemma 3.2. Let

$$
\left(\begin{array}{cc}
\kappa_{1} & * \\
0 & \kappa_{2}
\end{array}\right)
$$

be Jordan's normal form of $J$. Since $J$ is parallel, the eigenvalue $\kappa_{1}$ of $J$ is constant on $V$. Now by replacing $\alpha$ with $\alpha-\left(\operatorname{Re} \kappa_{1}\right) \omega$ we may assume that $\kappa_{1}$ is purely imaginary, and consequently $J^{2}$ has a nonpositive real eigenvalue $-\mu^{2}=\kappa_{1}^{2}$. If $L^{-} \subset E^{-}$denotes the eigenspace of the eigenvalue $-\mu^{2}$ of $J^{2}$, then $L^{-}$has the same dimension at each point of $V$ since $J$ is parallel: In other words $L^{-}$is a subbundle of $E^{-}$. However it is clear that the Euler class of $E^{-}$is nontrivial (In fact the restriction of $E^{-}$to a fiber of the unit tangent bundle $V \rightarrow M$ is isomorphic to the tangent bundle of the 2 -sphere). Hence $E^{-}$never contains a nontrivial proper subbundle. This especially implies that $L^{-}=E^{-}$, and eventually that $J^{2}=-\mu^{2}$. Moreover the constant $\mu$ should be different from zero: Otherwise there would occur a contradiction to the choice of $\alpha$. Finally replace $\alpha$ by $\alpha / \mu$. Then we get $J^{2}=-1$; i.e., $J$ is an almost complex structure of the stable bundle $E^{-}$. Together with Theorem 2.1 this proves Theorem 3.1.

\section{$\S 4$. Smoothness of the stable bundle of the frame flow}

The aim of the present section is to indicate an obstruction to the smoothness of the stable and unstable bundles of the frame flow on a certain negatively curved manifold as an application of results obtained so far. Let us begin with recalling the definition of the frame flow (cf. [Gre], [BP], [B1], [B2], [BG], [BK]). Suppose that $M$ is an oriented complete riemannian manifold of dimension $m+1$. Hereafter denote by $W$ the space of positively oriented orthonormal frames of $M$ : Each point of it is by definition an ordered $(m+1)$-tuple $\left(v_{0}, \ldots, v_{m}\right)$ of orthonormal tangent vectors of $M$ of positive orientation. As is well known, $P$ is a principal fiber bundle over $M$ with structure group $\mathrm{SO}(m+1)$. In addition by assigning the first entry $v_{0}$ to each $p=\left(v_{0}, \ldots, v_{m}\right) \in P$ we can define a projection of $P$ onto the unit tangent bundle $V$ of $M$, which provides $P$ with a structure of principal $\mathrm{SO}(m)$ bundle over $V$. Furthermore the geodesic flow $\varphi_{t}$ of $M$ defined on $V$ can be lifted to a smooth flow $\psi_{t}$ of $P$, called the frame flow of $M$, in the following way. For each 
$w=\left(v_{0}, \ldots, v_{m}\right) \in W$, first take the geodesic in $M$ whose initial velocity is the first entry $v_{0}$ of $w$, and next take the parallel translation of the frame $w$ along the geodesic: Then $\psi_{t}(w) \in W$ is defined to be the parallel translation of $w$ at the time $t$. Assume now that $M$ is closed and of negative curvature. Then the geodesic flow $\varphi_{t}$ of $M$ is known to be Anosov. Moreover it is known (cf. [BP]) that in this case the hyperbolicity of the geodesic flow also gives rise to a splitting of the tangent bundle of $W$;

$$
T W=F^{v e r}+F^{h o r} \text { with } F^{h o r}=F^{-}+F^{0}+F^{+},
$$

and the subbundles appearing in this splitting satisfy the following conditions: (i) $F^{v e r}$ is vertical with respect to the fibering $\pi: W \rightarrow V$, and hence is characterized by $F^{v e r}=\operatorname{Ker} d \pi$; meanwhile $F^{h o r}$ is horizontal with respect to $\pi$; (ii) $F^{0}$ is 1-dimensional and tangent to the orbits of the frame flow $\psi_{t}$; (iii) for $\eta^{-} \in F^{-}$ and $\eta^{+} \in F^{+}, d \psi_{t} \eta^{-}$and $d \psi_{t} \eta^{+}$contract exponentially as $t \rightarrow+\infty$ and $t \rightarrow-\infty$ respectively. Note that this splitting is continuous and unique, and especially the latter property also implies that the splitting is invariant under both the frame flow $\psi_{t}$ and the action of the structure group $\mathrm{SO}(m)$ on $W$.

Our purpose here is to show the following partial result supporting the plausibility of the conjecture on the differentiability of the horizontal bundle $F^{\text {hor }}$ which we have proposed in the introduction.

TheOREM 4.1. Suppose that $M$ is a closed 3-dimensional riemannian manifold of negative curvature whose geodesic flow satisfies the pinching condition $\Lambda / \lambda<2$. If the horizontal bundle $F^{\text {hor }}$ associated with the frame flow of $M$ is $C^{2}$-differentiable, then the geodesic flow of $M$ is homothetic to that of a certain closed hyperbolic manifold.

Our starting point is the simple observation that the horizontal bundle $F^{\text {hor }} \subset$ $T W$ can be regarded as a connection of the principal fiber bundle $W$ over $V$. In fact the existence of the splitting of $T W$ mentioned earlier will be proved in $\S 4.1$ based on this geometric idea. Moreover if $F^{\text {hor }}$ is smooth then its curvature is defined, and will play the central role in the proof of Theorem 4.1. In fact the proof will be done in $\S 4.2$ by projecting down the curvature to a differential form on the unit tangent bundle which is invariant under the geodesic flow and by applying the previous theorem 3.1.

4.1. First we want to show the existence of the horizontal subbundle $F^{\text {hor }}$ associated with the frame flow, which has been first established by Brin and Pesin $[\mathrm{BP}]$; however our approach adapted here is much more geometric than theirs. The proof given here as well as that of Brin-Pesin works even for a broader class of dynamical systems, called the extensions of Anosov systems by compact Lie groups, so we will prove the existence of the horizontal bundles for such systems. Let us 
begin by reviewing the definition of those dynamical systems. Let $W$ be a principal fiber bundle over a differentiable manifold $V$ whose structure group is a Lie group $G$. Then a smooth flow $\psi_{t}$ on $W$ is called a $G$-extension of a flow $\varphi_{t}$ on the base manifold $V$ if the following two conditions are satisfied: (i) $\pi \circ \psi_{t}=\varphi_{t} \circ \pi$, where $\pi$ is the projection of $W$ onto $V$; (ii) $\psi_{t} \circ R_{g}=R_{g} \circ \psi_{t}$, where $R_{g}(g \in G)$ denotes the action of the structure group $G$ on $W$ (from right). Obviously the frame flow of an $(m+1)$-dimensional riemannian manifold $M$ is a $\mathrm{SO}(m)$-extension of the geodesic flow of $M$. Now we can state what we are going to see in the present subsection.

Proposition 4.2. Suppose that $W$ is a principal fiber bundle over a closed differentiable manifold $V$ with a compact structure group $G$, and assume especially that a flow $\psi_{t}$ on $W$ is a $G$-extension of an Anosov flow $\varphi_{t}$ on $V$. Then there always exists a unique continuous connection $F^{\text {hor }}$ of $W$ which is adapted for $\psi_{t}$.

Here as usual by a continuous connection of the principal bundle $W$ is meant a continuous subbundle $F^{\text {hor }}$ of the tangent bundle $T W$ such that (i) $T W$ is the direct sum of $F^{\text {hor }}$ and the vertical subbundle $F^{v e r}=\operatorname{Ker} d \pi$, and that (ii) $F^{\text {hor }}$ is invariant under the action of the structure group $G$ on $W$. Furthermore a connection $F^{h o r}$ of $W$ is said to be adapted for the $G$-extension $\psi_{t}$, if (iii) $F^{h o r}$ is invariant under $\psi_{t}$ and if (iv) the vector field $\dot{\psi}$ on $W$ that generates the flow $\psi_{t}$ is a section of $F^{h o r}$. Hence in the proposition the derivative $d \pi$ of the projection $\pi: W \rightarrow V$ gives rise to a linear isomorphism between a fiber of $F^{\text {hor }}$ and a tangent space of $V$, and the Anosov splitting $T V=E^{-}+E^{0}+E^{+}$associated with the Anosov flow $\varphi_{t}$ on the base manifold $V$ yields a splitting $F^{h o r}=F^{-}+F^{0}+F^{+}$upstairs which satisfies the following conditions: (v) $F^{0}$ is 1-dimensional and spanned by the vector field $\dot{\psi}$ generating the flow $\psi_{t}$; (vi) each tangent vector in $F^{-}$(resp. $F^{+}$) contracts exponentially along the orbit of $\psi_{t}$ in the positive (resp. negative) direction. The connection $F^{\text {hor }}$ adapted for $\psi_{t}$, whose unique existence is guaranteed in the above proposition, will be called the Brin-Pesin connection here.

Proof of Proposition 4.2. It is well known that a connection of the principal bundle $W$ is equivalent to a $\mathfrak{g}$-valued 1-form $\Theta$ on $W$ such that $R_{g}^{*} \Theta=\operatorname{Ad}\left(g^{-1}\right) \circ$ $\Theta$, and that $\Theta\left(X^{*}\right)=X$ for any $X \in \mathfrak{g}$, where $\mathfrak{g}$ denotes the Lie algebra of the structure group $G$, and for each $X \in \mathfrak{g}, X^{*}$ denotes the vertical vector field on $W$, called a fundamental vector field, that is defined by $X^{*}(w)=(d / d t)_{t=0} R_{\exp t X}(w)$ at $w \in W$. Actually if $\Theta$ is such a $\mathfrak{g}$-valued 1 -form, which is usually called a connection 1-form, then at each point $w$ of $W \operatorname{Ker} \Theta_{w} \subset T_{w} W$ defines the fiber of the connection $F^{\text {hor }}$ at $w$. Conversely if $F^{h o r}$ is a connection, then the projection of the tangent bundle $T W$ to the vertical subbundle $F^{v e r}$ along $F^{h o r}$ is a connection 1 -form provided that the fiber of $F^{v e r}$ at each point of $W$ is identified with the Lie algebra $\mathfrak{g}$ by means of the fundamental vector fields. See for detail [KN], [St].

Now let $T V=E^{-}+E^{0}+E^{+}$be the Anosov splitting of the Anosov flow 
$\varphi_{t}$ on the base manifold $V$, and $\widehat{F}^{\delta}=d \pi^{-1}\left(E^{\delta}\right)(\delta=0, \pm)$ be the $\psi_{t}$-invariant continuous subbundles of $T W$ obtained by taking the preimages of the subbundles $E^{\delta}$ of $T V$ by the derivative $d \pi: T W \rightarrow T V$ of the projection $\pi: W \rightarrow V$. Clearly each $\widehat{F}^{\delta}$ contains the vertical bundle $F^{v e r}$. Furthermore if a connection $F^{h o r}$ on $W$ is given, then it induces the splitting $\widehat{F}^{\delta}=F^{v e r}+F^{\delta}$ of each $F^{\delta}$, where $F^{\delta}(\delta=0, \pm)$ are the components of the splitting $F^{\text {hor }}=F^{-}+F^{0}+F^{+}$ which comes out of the Anosov splitting $T V=E^{-}+E^{0}+E^{+}$as before. Conversely the splittings $\widehat{F}^{\delta}=F^{v e r}+F^{\delta}$ clearly determine the connection $F^{h o r}$. Finally note that each splitting $\widehat{F}^{\delta}=F^{v e r}+F^{\delta}$ is also equivalent to a $\mathfrak{g}$-valued 1 -form $\Theta^{\delta}$ on $\widehat{F}^{\delta}$ (or equivalently, a map $\widehat{F}^{\delta} \rightarrow \mathfrak{g}$ which is linear on each fiber of $\widehat{F}^{\delta}$ ) such that $R_{g}^{*} \Theta^{\delta}=\operatorname{Ad}\left(g^{-1}\right) \circ \Theta^{\delta}$, and that $\Theta^{\delta}\left(X^{*}\right)=X$ for $X \in \mathfrak{g}$. Actually this identification can be seen in the same way as before. We call such $\Theta^{\delta}$ a connection 1-form of $\widehat{F}^{\delta}$. What we have to prove to conclude the proposition is consequently that there are unique $\psi_{t}$-invariant continuous connection forms $\Theta_{B P}^{\delta}$ of $\widehat{F}^{\delta}(\delta=0, \pm)$ with $\Theta_{B P}^{\circ}(\dot{\psi})=0$.

In the case of $\delta=0$, this is trivial since $\Theta_{B P}^{\circ}$ is characterized by the condition that $\Theta_{B P}^{\circ}(\dot{\psi})=0$. So consider the case of $\delta=-$ (The other case $\delta=+$ can be dealt with in the same way, and will be omitted). Let $\Theta_{0}^{-}$and $\Theta_{1}^{-}$be arbitrary continuous connection forms of $\widehat{F}^{-}$. Then their difference $\Delta=\Theta_{1}^{-}-\Theta_{0}^{-}$is a $\mathfrak{g}-$ valued 1 -form on $\widehat{F}^{-}$which vanishes on $F^{v e r} \subset \widehat{F}^{-}$. Hence $\Delta$ can be regarded as a $\mathfrak{g}$-valued 1-form on $\widehat{F}^{-} / F^{v e r}=\pi^{*} E^{-}$, where $\pi^{*} E^{-}$denotes the pull-back of the stable bundle $E^{-}$through the projection $\pi$. Thus in $\psi_{t}^{*} \Theta_{1}^{-}-\psi_{t}^{*} \Theta_{0}^{-}=\psi_{t}^{*} \Delta$ the last $\psi_{t}^{*}$ is the induced action of $\psi_{t}$ on $\left(\widehat{F}^{-} / F^{v e r}\right)^{*} \otimes \mathfrak{g}=\left(\pi^{*} E^{-}\right)^{*} \otimes \mathfrak{g}$, and therefore is essentially equal to $\varphi_{t}^{*} \otimes i d$. Clearly the induced action $\varphi_{t}^{*}$ of the Anosov flow $\varphi_{t}$ on $\left(\pi^{*} E^{-}\right)^{*}$ is uniformly contracting for $t<0$. Consequently it follows that the $C^{0}$-norm $\left\|\psi_{t}^{*} \Theta_{1}^{-}-\psi_{t}^{*} \Theta_{0}\right\|=\left\|\psi_{t}^{*} \Delta\right\|$ tends to zero exponentially as $t \rightarrow-\infty$ : In other words the induced flow on the Banach space of continuous connection forms on $\widehat{F}^{-}$is uniformly contracting. Therefore it has a unique fixed point $\Theta_{B P}^{-}$, that is clearly a unique $\psi_{t}$-invariant continuous connection form of $F^{-}$.

4.2. Here is a proof of Theorem 4.1. Take $M$ as in the theorem, and assume especially that the Brin-Pesin connection $F^{h o r}$ of the frame flow is $C^{2}$-differentiable. Then we can think of the curvature form $\Omega_{B P}$ of the Brin-Pesin connection $F^{h o r}$, which is by definition a $C^{1}$-differentiable $\psi_{t}$-invariant $\mathfrak{g}$-valued 2 -form on $W$ satisfying $R_{g}^{*} \Omega_{B P}=\operatorname{Ad}\left(g^{-1}\right) \circ \Omega_{B P}$ and $\left.\eta^{v e r}\right\lrcorner \Omega_{B P}=0$ for $\eta^{v e r} \in F^{v e r}$. Furthermore in this case the structure group $G=\mathrm{SO}(2)$ is 1-dimensional, and therefore $\Omega_{B P}$ descends to an (R-valued) 2 -form $\omega_{B P}$ on $V$ via the projection $W \rightarrow V$. It is clear that $\omega_{B P}$ is a $C^{1}$-differentiable $\varphi_{t}$-invariant transverse 2 -form; that is, $\omega_{B P}$ is an element of the sapce $\Omega_{\varphi_{t}}^{*}(V)$ which has been introduced in the previous section. Recall now that $\Omega_{\varphi_{t}}^{*}(V)$ always contains the exterior derivative $\omega=d \theta$ of the canonical contact form $\theta$. Thus according to Theorem 3.1 it is sufficient to prove the following lemma 
to complete the proof of Theorem 4.1.

Lemma 4.3. $\omega_{B P}$ is linearly independent of $\omega$.

Proof. Let $\Theta=\pi^{*} \theta$ be the pull-back of the canonical contact form $\theta$ on $V$ to $W$ through the projection $\pi: W \rightarrow V$. Then it is clear that $d \Theta=\pi^{*} \omega$. Meanwhile let $\Theta_{B P}$ be the connection form of the Brin-Pesin connection $F^{\text {hor }}$. By definition $\Theta_{B P}$ is an $\mathfrak{s o}(2)$-valued 1-form on $W$, and the curvature form $\Omega_{B P}$ of the Brin-Pesin connection is as in general given by $\Omega_{B P}=d \Theta_{B P} \circ h$ with $h: T W \rightarrow F^{h o r}$ being the horizontal projection, or more precisely $\Omega_{B P}(\eta, \zeta)=d \Theta_{B P}(h \eta, h \zeta)$ for $\eta, \zeta \in T W$. However especially in our case the 1-dimensionality of the fibers of $W \rightarrow V$ implies that $d \Theta_{B P} \circ h=d \Theta_{B P}$. Thus it follows that $d \Theta_{B P}=\Omega_{B P}=\pi^{*} \omega_{B P}$. Now assume, contrary to the lemma, that $\omega_{B P}$ is proportional to $\omega$; i.e., that there is a constant $\kappa$ such that $\omega_{B P}=\kappa \omega$. Then the identities obtained above imply that $d \Theta_{B P}=\kappa d \Theta$. On the other hand let $c^{1}$ be a fiber of $W \rightarrow V$, which is of course homeomorphic to the circle $\mathrm{SO}(2)$. Obviously $c^{1}$ is contained in a certain fiber of $W \rightarrow M$ which is homeomorphic to $\mathrm{SO}(3)$. However $H_{1}(\mathrm{SO}(3) ; \mathbb{R})$, the 1-dimensional homology of $\mathrm{SO}(3)$ with real coefficients, is trivial, and this in particular means that $c^{1}$ is homologous to zero. In other words we can find a (smooth) 2-chain $c^{2}$ (lying in a fiber of $W \rightarrow M$ ) that is bounded by $c^{1}$. Now according to Stokes' theorem and the fact that $\Theta \mid F^{v e r}=0$ we have

$$
0=\int_{c^{2}} d\left(\Theta_{B P}-\kappa \Theta\right)=\int_{c^{1}}\left(\Theta_{B P}-\kappa \Theta\right)=\int_{c^{1}} \Theta_{B P} \neq 0
$$

which is clearly a contradiction.

\section{References}

[ A ] D.V. Anosov, Geodesic flows on closed riemannian manifolds with negative curvature (English translation), Proc. Steklov Inst. Math., 90 (1969), 1-235.

[ $\mathrm{B}^{3}$ ] W. Ballmann, M. Brin and K. Burns, On the differentiability of horocycles and horocycle foliations, J. Differential Geometry, 26 (1987), 337-347.

[BFL] Y. Benoist, P. Foulon and F. Labourie, Flots d'Anosov a distributions stable et instable differentiables, J. Amer. Math. Soc., 5 (1992), 33-74.

[ B1 ] M.I. Brin, Topological transitivity of one class of dynamic systems and flows of frames on manifolds of negative curvature, Functional Anal. Appl., 9 (1975), 8-16.

[ B2 ] M.I. Brin, The topology of group extensions of $C$ systems, Math. Notes, 18 (1975), 858-864.

[ BG ] M.I. Brin and M. Gromov, On the ergodicity of frame flows, Invent. Math., 60 (1980), 1-7.

[ BK ] M.I. Brin and H. Karcher, Frame flows on manifolds with pinched negative curvature, Compositio Math., 52 (1984), 275-297.

[ BP ] M.I. Brin and Ja. B. Pesin, Partially hyperbolic dynamical systems (English translation), Math., USSR-Izv., 8 (1974), 177-218.

[ EO ] P. Eberlein and B. O'Neill, Visibility manifolds, Pacific J. Math., 46 (1973), 45-109. 
[ F ] R. Feres, Geodesic flows on manifolds of negative curvature with smooth horospheric foliations, Thesis, California Institute of Technology, 1989.

[FK1] R. Feres and A. Katok, Invariant tensor fields of dynamical systems with pinched Lyapunov exponents and rigidity of geodesic flows, preprint.

[FK2] R. Feres and A. Katok, Anosov flows with smooth foliations and rigidity of geodesic flows in three-dimensional manifolds of negative curvature, preprint.

[ FIK ] L. Flaminio and A. Katok, Rigidity of symplectic Anosov diffeomorphisms on low dimensional tori, preprint.

[ Gh ] E. Ghys, Flots d'Anosov dont des feuilletages stable sont differentiables, Ann. Sci. École Norm. Sup., 20 (1987), 250-270.

[ Gre ] L.W. Green, The generalized geodesic flow, Duke Math. J., 41 (1974), 115-126; Correction, ibid., 42 (1975), 381.

[ Gro ] M. Gromov, Hyperbolic manifolds, groups and actions, in "Rieman Surfaces and Related Topics," Ann. of Math. Studies, no. 97, Princeton Univ. Press, Princeton, 1981, pp. 183-213.

[ $\mathrm{Hr}$ ] P. Hartman, On isometries and on a theorem of Liouville, Math. Z., 69 (1958) 202-210.

[ Hs ] B. Hasselblatt, Regularity of the Anosov splitting and a new description of the Margulis measure, Thesis, Caltech, 1989.

[HP1] M.W. Hirsch and C.C. Pugh, Stable manifolds and hyperbolic sets, in Proc. Sympos. Pure Math., vol. 14, Amer. Math. Soc., Providence, 1970, pp. 133-163.

[HP2] M.W. Hirsch and C.C. Pugh, Smoothness of horocycle foliations, J. Differential Geometry, 1 (1975), 225-238.

[ HK ] S. Hurder and A. Katok, Differentiability, rigidity and Godbillon-Vey classes for Anosov flows, preprint.

[ K1 ] M. Kanai, Geodesic flows of negatively curved manifolds with smooth stable and unstable foliations, Ergod. Th. \& Dynam. Sys., 8 (1988), 215-239.

[ K2 ] M. Kanai, Tensorial ergodicity of geodesic flows, in "Geometry and Analysis on Manifolds," Springer Lecture Notes in Math., no. 1339, 1988, pp. 142-157.

[ K3 ] M. Kanai, A pinching theorem for cusps of negatively curved manifolds, Proc. Amer. Math. Soc, 107 (1989), 777-783.

[ $\mathrm{Kr}$ ] H. Karcher, Riemannian center of mass and molifier smoothing, Comm. Pure Appl. Math., 30 (1977), 509-541.

[ Kt ] A. Katok, Entoropy and closed geodesics, Ergod. Th. \& Dynam. Sys., 2 (1982), 339-367.

[ KN ] S. Kobayashi and K. Nomizu, Foundations of Differential Geometry, vol. I, Intersience, New York, 1963.

[ L ] J. Lelong-Ferrand, Geometric interpretations of scalar curvature and regularity of conformal homeomorphisms, in "Differential Geometry and Relativity," Math. Phys. and Appl. Math., vol. 3, Reidel, Dordrecht, 1976, pp. 91-105.

[ M1 ] G.D. Mostow, Quasi-conformal mappings in $n$-space and the rigidity of hyperbolic space forms, Publ. Math. IHES, 34 (1968), 53-104.

[ M2 ] G.D. Mostow, Strong Rigidity of Locally Symmetric Spaces, Ann. of Math. Studies, no. 78, Princeton Univ. Press, Princeton, 1973.

[St ] S. Sternberg, Lectures on Differential Geometry, Chelsea, New York, 1964

[ S1 ] D. Sullivan, On the ergodic theory at infinity of an arbitrary discrete group of hyperbolic motions, in "Riemann Surfaces and Related Topics," Ann. of Math. Studies, no. 97, Princeton Univ. Press, Princeton, 1981, pp. 465-496.

[ S2 ] D. Sullivan, Quasiconformal homeomorphisms in dynamics, topology and geometry, in Proceedings of ICM 1986. 
[ Th ] W. Thurston, The geometry and topology of three-manifolds, Lecture Notes, Princeton Univ., 1979.

[ Tu ] P. Tukia, On two-dimensional quasiconformal groups, Ann. Acad. Sci. Fenn., 5 (1980), $73-78$.

Department of Mathematics

KEIO UNIVERSITY

YOKOHAMA 223, JAPAN 